Ergod. Th. \& Dynam. Sys. (1987), 7, 49-72

Printed in Great Britain

\title{
Semi-rigidity of horocycle flows over compact surfaces of variable negative curvature
}

\author{
J. FELDMAN AND D. ORNSTEIN \\ Department of Mathematics, University of California, Berkeley, CA 94720, USA; \\ Department of Mathematics, Stanford University, Stanford, CA 94305, USA
}

(Received 5 November 1985 and revised 18 April 1986)

\begin{abstract}
Let $g$ be the geodesic flow on the unit tangent bundle of a $C^{3}$ compact surface of negative curvature. Let $\mu$ be the $g$-invariant measure of maximal entropy. Let $h$ be a uniformly parametrized flow along the horocycle foliation, i.e., $g h_{\mathrm{e} r s}=$ $h_{r_{s}} g_{r}$; such a flow exists, leaves $\mu$ invariant, and is unique up to constant scaling of the parameter (Margulis). We show that any measure-theoretic conjugacy: $(h, \mu) \rightarrow\left(h^{\prime}, \mu^{\prime}\right)$ is a.e. of the form $h_{s_{0}}^{\prime} \theta$, where $\theta$ is a homeomorphic conjugacy: $g \rightarrow g^{\prime}$. Furthermore, any homeomorphic conjugacy $g \rightarrow g^{\prime}$ must be a $C^{1}$ diffeomorphism.
\end{abstract}

\section{Introduction}

In the last few years, M. Ratner has proven a series of 'rigidity' theorems for classical horocycle flows; see [R4] for a survey of these. In the present paper we obtain a partial generalization, in a geometric context, of her central result in [R1].

Ratner's main theorem is most easily described algebraically. Here is a slight specialization of it. Let $\mathscr{G}=\operatorname{PSL}(2, \mathbb{R})=\operatorname{SL}(2, \mathbb{R}) /\{ \pm$ identity $\}$. Let $\Gamma$ be a lattice subgroup of $\mathscr{G}$, i.e. $\Gamma$ is discrete and $\Gamma \backslash \mathscr{G}$ has an invariant probability measure $\mu$. On general grounds, $\mu$ is unique and is equivalent to the image of the right Haar measure on $\mathscr{G}$. Here are three important flows on $\Gamma \backslash \mathscr{G}$ :

(1) the geodesic flow $g$, given by

$$
g_{r}(\Gamma \gamma)=\Gamma \gamma\left(\begin{array}{cc}
e^{r / 2} & 0 \\
0 & e^{-r / 2}
\end{array}\right)
$$

(2) the expanding horocycle flow $h$, given by

$$
h_{s}(\Gamma \gamma)=\Gamma \gamma\left(\begin{array}{ll}
1 & 0 \\
s & 1
\end{array}\right)
$$

(3) the contracting horocycle flow $k$, given by

$$
k_{t}(\Gamma \gamma)=\Gamma \gamma\left(\begin{array}{ll}
1 & t \\
0 & 1
\end{array}\right)
$$

The terminology arises from the connection with geometry, which will be discussed briefly below. The following commutation relations hold:

$$
g_{r} h_{s} g_{-r}=h_{e^{r} s} \text { and } g_{r} k_{t} g_{-r}=k_{e^{-r}} \text {. }
$$


The flows obviously leave $\mu$ invariant. If $\Gamma^{\prime}=\gamma_{0} \Gamma \gamma_{0}^{-1}$, let

$$
\theta_{\gamma_{0}}(\Gamma \gamma)=\gamma_{0} \Gamma \gamma=\Gamma^{\prime} \gamma_{0} \gamma \in \Gamma^{\prime} \backslash \mathscr{G}
$$

$\theta_{\gamma_{0}}$ carries $g_{r}, h_{s}, k_{t}$ to the corresponding $g_{r}^{\prime}, h_{s}^{\prime}, k_{t}^{\prime}$ on $\Gamma^{\prime} \backslash \mathscr{G}$, and, because of unimodularity of $\mathscr{G}$, carries $\mu$ to $\mu^{\prime}$. In particular, $\theta_{\gamma_{0}}$ is a measure-theoretic conjugacy: $\left(h_{1}, \mu\right) \rightarrow\left(h_{1}^{\prime}, \mu^{\prime}\right)$. (Conjugacies in this paper are always invertible.) Conversely:

(1.1) THEOREM (Ratner [R1]). Let $\phi$ be a measure-theoretic conjugacy: $\left(h_{1}, \mu\right) \rightarrow$ $\left(h_{1}^{\prime}, \mu^{\prime}\right)$. Then there exists $\gamma_{0} \in \mathscr{G}$ and a real number $s_{0}$ such that $\phi=h_{s_{0}}^{\prime} \theta_{\gamma_{0}} \quad \mu$-a.e.

The theorem may be interpreted geometrically. We shall do so here only for the case when $\Gamma \backslash \mathscr{G}$ is compact, since our generalization involves only this case. Let $S$ be a $C^{3}$ compact orientable surface of negative curvature, $U(S)$ its unit tangent bundle. There is a natural flow on $U(S)$, again called the geodesic flow and again written $g$. It has a unique smooth invariant measure. It is a $C^{2}$ Anosov flow; thus there exist a foliation $\mathscr{H}$ into expanding curves and a foliation $\mathscr{K}$ into contracting curves. We shall assume the metric of $S$ to be so normalized, and therefore the speed of $g$ to be so scaled, that $g$ has topological entropy 1 .

Now consider the special case when $S$ has constant negative curvature. Then the aforementioned normalization has the effect of making the curvature -1 ; and the $U(S)$ which arise from various such $S$ are naturally diffeomorphic to the $\Gamma \backslash \mathscr{G}$ arising from various cocompact $\Gamma$. Each of these diffeomorphisms carries $g$ on $\Gamma \backslash \mathscr{G}$ to $g$ on the corresponding $U(S)$, which is our reason for using the same notation and terminology; and carries $h$ and $k$ on $\Gamma \backslash \mathscr{G}$ to flows along the curves of $\mathscr{H}$ and $\mathscr{K}$. The image flows may be characterized geometrically on $U(S)$, up to certain unavoidable ambiguities. Let us again call the image flows $h$ and $k$, and the image measure $\mu$. Then theorem 1.1 translates into this:

(1.2) THEOREM. If $\phi: U(S) \rightarrow U\left(S^{\prime}\right)$ is a measure-theoretic conjugacy of $\left(h_{1}, \mu\right)$ with $\left(h_{1}^{\prime}, \mu^{\prime}\right)$, then $\phi=h_{s_{0}}^{\prime} \theta$ a.e., where $\theta$ is the lifting of some isometry: $S \rightarrow S^{\prime}$ to the unit tangent bundles.

It is this geometric form of the result which we shall generalize.

In the variable curvature case, in addition to the geodesic flow $g$, there are again choices of flows $\bar{h}$ and $\bar{k}$ along $\mathscr{H}$ and $\mathscr{K}$ which satisfy appropriate commutation relations with $g$. The existence of such parametrizations of the curves of $\mathscr{H}$ and $\mathscr{K}$ was shown by Margulis [Ma]; see also Marcus [M1]; they are essentially unique. In the constant curvature case they coincide with $h$ and $k$; but in the general case they are not even $C^{1}$, although they are continuous. One effect of the 'normalization' assumption is to scale the commutation relations properly. There is a unique probability measure $\mu$ invariant under $\bar{h}$. It is then natural to ask (see [R1]) whether something like (1.2) holds. Our result in this context is that for any measure-theoretic isomorphism $\phi$ from $(\bar{h}, \mu)$ to $\left(\bar{h}, \mu^{\prime}\right)$ there is some $s_{0}$ such that $\phi=\bar{h}_{\text {a.e. }}^{\prime} \theta$, where $\theta$ is a homeomorphic conjugacy of $g$ with $g^{\prime}$. This is of course much weaker than saying that $\theta$ is the lifting of an isometry between $S$ and $S^{\prime}$. But if either $M$ or $M^{\prime}$ is of constant curvature, then it easily follows that $\theta$ is the lifting of an isometry. Incidentally, there is a natural Riemannian metric on any unit tangent bundle, and 
$\theta$ will be the lifting of an isometry if and only if it is an isometry for this metric (theorem 6.1).

Our main result, corollary 4.6 , is obtained in the possibly more general context where $g$ and $g^{\prime}$ are $C^{2}$ Anosov flows on compact orientable 3-manifolds $M$ and $M^{\prime}$, and satisfy certain natural conditions which are automatic in the 'geometric' case. It turns out that $\theta$ is actually more than just a homeomorphism: it is in fact a $C^{1}$ diffeomorphism (theorem 5.2).

Here is the strategy for the proof of the main result. First, in $\$ 2$, we get uniform estimates on the non-integrability of expanding and contracting curves of $(g, M)$ : this is proposition 2.6. Switching to Margulis's uniform parametrization, which we indicate by writing $\bar{h}$ and $\bar{k}$, this leads to proposition 3.9 , the statement that $\bar{h}$ satisfies Ratner's ' $\mathscr{H}_{p}$ property' [R3], which immediately gives corollary 3.10, the generalization to the present context of Ratner's 'Basic Lemma' of [R1]. This is precisely what was used there to prove, for constant curvature, that $\phi$ is of the form $\bar{h}_{s_{0}}^{\prime} \psi$, where $\psi$ carries $(g, \mu)$ to $\left(g^{\prime}, \mu^{\prime}\right)$ as a measure-theoretic conjugacy; and the argument works equally well for variable curvature. Finally, it must be shown that $\psi$ carries $(\bar{k}, \mu)$ to $\left(\bar{k}^{\prime}, \mu^{\prime}\right)$; this is proposition 4.1. Our proof is quite different from the original argument in [R1] which relied on the algebraic nature of the constant curvature case. A significant simplification of our original argument has been found by $\mathrm{L}$. Flaminio; it is in fact Flaminio's simplification which will be given here. In $\S 6$ we describe two results relating to the problem of getting from a homeomorphic conjugacy of geodesic flows to an isometry of surfaces. A. Weinstein provided the main argument for the first of these, and the second is due to J. Smillie. We also obtain, using a result of $\mathrm{A}$. Katok, an isometry result for the case where the homeomorphic conjugacy arises from a small conformal change in a fixed Riemannian metric.

We owe thanks to various people; especially to C. Croke, for teaching one of us enough geometry, during several afternoons at the Upper Level Café, to make it possible to carry out $\S 2$; to L. Flaminio for his careful reading of our arguments, and for allowing us to inciude his simplification of one of them; to $M$. Ratner for several valuable discussions, and especially for pointing out errors at various stages; and to $\mathrm{A}$. Weinstein for proving the non-trivial part of proposition 6.1.

This paper was written with partial support from NSF grants MCS-83-07086 and MCS-81-07092, and from the Mathematical Sciences Research Institute in Berkeley. The first-named author also wishes to thank the School of Mathematics of the University of New South Wales for its assistance and hospitality.

\section{A local limit for good Anosov flows}

(2.1) Lemma. Let $M$ be a compact $C^{2}$ manifold, and $B, C$ two $C^{1}$ vector fields generating flows $b, c$. Then for any $\phi \in C^{2}(M)$,

$$
B(C \phi)(x)-C(B \phi)(x)=\lim _{s, t \rightarrow 0} \frac{1}{s t}\left[\phi\left(c_{t} b_{s} x\right)-\phi\left(b_{s} c_{t} x\right)\right]
$$

uniformly in $x$. 
Proof.

$$
\begin{aligned}
\phi\left(c_{t} b_{s} x\right)-\phi\left(b_{s} c_{t} x\right)= & \int_{0}^{t} C \phi\left(c_{t^{\prime}} b_{s} x\right) d t^{\prime}-\int_{0}^{t} C \phi\left(c_{t^{\prime}} x\right) d t^{\prime} \\
& -\int_{0}^{s} B \phi\left(b_{s^{\prime}} c_{t} x\right) d s^{\prime}+\int_{0}^{s} B \phi\left(b_{s} x\right) d s^{\prime} .
\end{aligned}
$$

Write $\psi$ for $C \phi$. Then $\psi \in C^{1}(M)$, so the map $(t, x) \mapsto \psi\left(c_{t} x\right)$ is in $C^{1}(\mathbb{R} \times M)$, and $(t, x) \mapsto B\left(\psi \circ c_{t}\right)(x)$ is in $C^{0}(\mathbb{R} \times M)$, and $(t, s, x) \mapsto B\left(\psi \circ c_{t}\right) b_{s}(x)$ is in $C^{0}(\mathbb{R} \times \mathbb{R} \times M)$. Then

$$
\lim _{s, t \rightarrow 0} B\left(\psi \circ c_{t}\right)\left(b_{s} x\right)=B \psi(x),
$$

uniformly in $x$.

Now:

$$
\begin{aligned}
\int_{0}^{t} C \phi\left(c_{t^{\prime}} b_{s} x\right) d t^{\prime}-\int_{0}^{t} C \phi\left(c_{t^{\prime}} x\right) d t^{\prime} & =\int_{0}^{t}\left(\left(\psi \circ c_{t^{\prime}}\right)\left(b_{s} x\right)-\psi \circ c_{t^{\prime}}(x)\right) d t^{\prime} \\
& =s \int_{0}^{t} B\left(\psi \circ c_{t^{\prime}}\right)\left(b_{s_{1}} x\right) d t
\end{aligned}
$$

where $s_{1}$ is some point (depending on $x$ and $t^{\prime}$ ) beween 0 and $s$; this is just the Mean Value theorem applied to $s \mapsto \psi \circ c_{t}\left(b_{s} x\right)$. Dividing by $s t$ gives

$$
\frac{1}{t} \int_{0}^{t} B\left(\psi \circ c_{t^{\prime}}\right)\left(b_{s_{1}} x\right) d t^{\prime}=\frac{1}{t} \int_{0}^{t}\left[B\left(\psi \circ c_{t^{\prime}}\right)\left(b_{s_{1}} x\right)-B(C \phi)(x)\right] d t^{\prime}+B(C \phi)(x) .
$$

The integrand $\rightarrow 0$, uniformly in $x$ as $s$ and $t \rightarrow 0$.

A similar argument, interchanging $B$ and $C$, gives

$$
\lim _{s, t \rightarrow 0} \frac{1}{s t} \int_{0}^{s}\left[B \phi\left(b_{s^{\prime}}\left(c_{t} x\right)\right) d s^{\prime}-\int_{0}^{s} B \phi\left(b_{s^{\prime}} x\right) d s^{\prime}\right]=C(B \phi)(x),
$$

uniformly in $x$. Subtracting gives the result.

Now let $G, H, K$ be nowhere vanishing vector fields on $M$, meeting transversally, and with the Lie bracket $[G, H]$ spanned by $H$ and $[G, K]$ by $K$. Then the orbits of $g$ and $h$ span a 2-dimensional foliation, as do those of $g$ and $k$.

$g, h$ and $k$ meet transversally, and so provide local coordinates (in six different ways, depending on the chosen order of application: $(r, s, t) \mapsto g_{r} h_{s} k_{t} x$, etc.). Utilizing this, we see that if $s$ and $t$ are sufficiently small then, for each $x \in M$, there are unique small $\rho, \sigma, \tau$, such that $g_{\rho} k_{\tau} h_{s} x=h_{\sigma} k_{t} x . \sigma$ has the same sign as $s$, and $\tau$ as $t$.

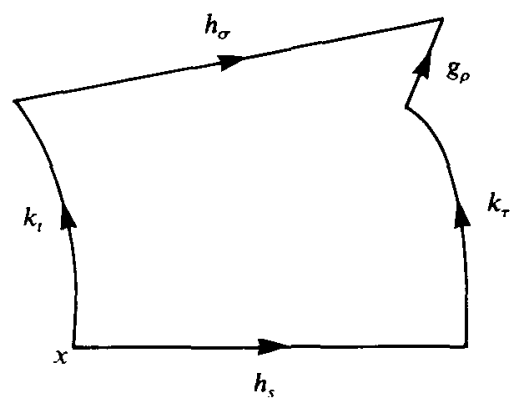

FIGURE 1 
$\tau$ is defined by: $k_{r} h_{s} x$ is the point where the local contracting curve through $h_{s} x$ first meets the local weakly expanding surface through $k_{t} x ; \sigma$ is defined analogously. $\rho, \sigma$, and $\tau$ are of course functions of $x, s$ and $t$, defined for all sufficiently small $s$, $t$ and all $x, \sigma$ has the same sign as $s$, and $\tau$ as $t$.

(2.2) LEMMA. For $s$ and $t$ sufficiently small, the functions $|\sigma(x, s, t)-s|,|\tau(x, s, t)-t|$, and $|\rho(x, s, t)|$ are all $\leq$ const. $|s t|$.

Proof. Let $d$ be the distance induced by a smooth metric on $M$. From lemma 2.1, there is for each bound $\Delta^{\prime}>0$ some $A^{\prime}>0$ so that if $|s|,|t|<\Delta^{\prime}$ then $d\left(h_{s} k_{t} x\right.$, $\left.k_{\mathrm{t}} h_{s} s\right) \leq A^{\prime}|s t|$ for all $x \in M$. By transversality of $H, K, G$ and compactness of $M$, there is some $\Delta<\Delta^{\prime}$ and some $A>A^{\prime}$ such that if $|s|,|t|,|r|<\Delta$ then, for all $y \in M$,

$$
\frac{1}{A}<\frac{d\left(k_{t} g_{r} h_{s} y, y\right)}{\sqrt{t^{2}+r^{2}+s^{2}}} .
$$

Let $s, t$ be small enough that $s, t, \sigma-s, \tau-s$, and $\rho$ are all smaller than $\Delta$; then, since by definition $k_{t-\tau} g_{-\rho} h_{\sigma-s}\left(h_{s} k_{t} x\right)=k_{t} h_{s} x$ (see figure 2), one has

$$
\begin{aligned}
\sqrt{(\sigma-s)^{2}+\rho^{2}+(\tau-t)^{2}} & <\operatorname{Ad}\left(k_{t-\tau} g_{-\rho} h_{\sigma-s}\left(h_{s} k_{t} x\right), h_{s} k_{t} x\right) \\
& =\operatorname{Ad}\left(k_{t} h_{s} x, h_{s} k_{t} x\right) \leq A^{2}|s t| .
\end{aligned}
$$

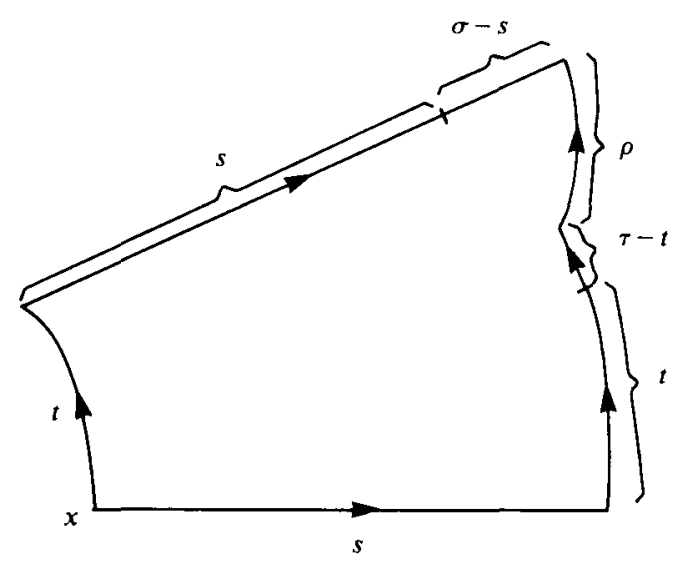

FIGURE 2

Now $[K, H]$ may be written as $a G+b H+c K$, with $a, b, c \in C^{0}(M)$.

(2.3) LEMMA. $\lim _{s, t \rightarrow 0} \rho / s t=a, \lim _{s, t \rightarrow 0}(\sigma-s) / s t=b$, and $\lim _{s, t \rightarrow 0}(t-\tau) / s t=c$, all uniformly on $M$.

Proof. For $\phi \in C^{2}(M)$,

$$
\phi\left(h_{\sigma} k_{t} \cdot\right)-\phi\left(k_{\tau} h_{s} \cdot\right)=\phi\left(g_{\rho} k_{\tau} h_{s} \cdot\right)-\phi\left(k_{r} h_{s} \cdot\right)=\rho G \phi\left(g_{\rho_{1}} k_{\tau} h_{s} \cdot\right),
$$

where $\rho_{1}$ lies between 0 and $\rho$. This equals

$$
\rho G \phi+\rho\left[G \phi\left(g_{\rho_{1}} k_{\tau} h_{s} \cdot\right)-G \phi\right] .
$$

But $|\rho|<$ const. $|s t|$, while $G \phi\left(g_{\rho_{1}} k_{\tau} h_{s} \cdot\right)-G \phi$ is small when $s$ and $t$ are small, so we get $\rho G \phi+o(s, t) s t$, where $o(s, t) \rightarrow 0$ as $s, t \rightarrow 0$. Meanwhile, $\phi\left(h_{\sigma} k_{t} \cdot\right)-\phi\left(k_{\tau} h_{s} \cdot\right)$ 
may be written as

$$
\begin{gathered}
\left(\phi\left(h_{s} k_{t} \cdot\right)-\phi\left(k_{t} h_{s} \cdot\right)\right)+\left(\phi\left(h_{\sigma} k_{t} \cdot\right)-\phi\left(h_{s} k_{t} \cdot\right)\right)-\left(\phi\left(k_{\tau} h_{s} \cdot\right)-\phi\left(k_{t} h_{s} \cdot\right)\right) \\
=[K, H] s t+o(s, t) s t+\int_{s}^{\sigma} H \phi\left(h_{s^{\prime}} k_{t} \cdot\right) d s^{\prime}-\int_{t}^{\tau} K \phi\left(k_{t^{\prime}} h_{s} \cdot\right) d t^{\prime},
\end{gathered}
$$

using lemma 2.1. Now,

$$
\begin{aligned}
\int_{s}^{\sigma} H \phi\left(h_{s^{\prime}} k_{t} \cdot\right) d s^{\prime} & =(\sigma-s) H \phi\left(h_{s_{1}} k_{t} \cdot\right) \\
& =(\sigma-s)\left(H \phi\left(h_{s_{1}} k_{t} \cdot\right)-H \phi\right)+(\sigma-s) H \phi \\
& =(\sigma-s) H \phi+o(s, t) s t .
\end{aligned}
$$

All this is uniform on $M$. Similarly,

$$
\int_{t}^{\tau} K \phi\left(k_{t^{\prime}} h_{s} \cdot\right) d t^{\prime}=(\tau-t) K \phi+o(s, t) s t
$$

So

$$
[K, H]=\frac{\rho}{s t} G+\frac{\sigma-s}{s t} H+\frac{t-\tau}{s t} K+o(s, t)
$$

uniformly in $M$.

(2.4) Definition. Now let $G$ be the vector field of a topologically transitive $C^{2}$ Anosov flow $g$, and $H, K$ nowhere vanishing expanding and contracting vector fields, respectively. We assume still that $H$ and $K$ are $C^{1}$. We also assume that $[K, H]$ is not identically zero. Such a $g$ we call a good Anosov flow.

The assumption $[K, H]$ not identically zero is equivalent to saying $g$ is not a suspension; and also that, if $\omega$ is the differential form defined by setting $\omega(H)=\omega(K)=0$ and $\omega(G)=1$, then $\omega \wedge d \omega$ is not identically zero. See Marcus $[\mathbf{M}]$ and Plante $[\mathbf{P}]$ for further discussion.

Finally, it should be noted that the geodesic flow on the unit tangent bundle of a compact $C^{3}$ surface of negative curvature is a good Anosov flow, and in fact this is the main case we have in mind.

We define a $C^{2}$ coordinate measure to be a finite measure on $M$ which locally looks like Lebesgue measured in appropriate $C^{2}$ coordinate charts. Such measures obviously exist, and any two differ by multiplication by a non-vanishing $C^{2}$ function. The following is just a more complete statement of a results in Plante [P].

(2.5) Proposition. A good Anosov flow has an invariant measure with continuous non-vanishing density with respect to any $C^{2}$ coordinate measure.

Proof (sketch). Following Plante, the aforementioned $\omega \wedge d \omega$ is an invariant continuous 3-form on $M$, and by assumption is not identically zero. So it gives rise to an absolutely continuous invariant measure $\lambda$ on $M$. By Anosov [A], this measure must actually be equivalent to any local $C^{2}$ coordinate measure; and $g$ is ergodic for $\lambda$. Then by Livčic and Sinai $[\mathbf{L}-\mathbf{S}], \lambda$ has a non-vanishing density with respect to any $C^{2}$ coordinate measure. 
(2.6) Corollary. a is never zero.

Proof. $\omega \wedge d \omega(G, H, K)$ may be computed to be the function $a$. Since $G, H, K$ span the tangent space at each point, and the measure associated with $\omega \wedge d \omega$ has non-vanishing density, $a$ is never 0 .

Summing up, we have:

(2.7) Proposition. $\rho(x, s, t) /$ st converges uniformly in $x$ as $s, t \rightarrow 0$, to a non-zero continuous function $a(x)$.

(2.8) Definition. We say $\{G, H, K\}$ is properly oriented if $a>0$. (This can always be achieved by reversing the direction of $K$ if necessary.)

(2.9) Lemma. If $\{G, H, K\}$ is properly oriented, then for fixed $t \neq 0$ and sufficiently small, $\rho(x, s, t)$ is monotonic in $s$ for sufficiently small $s:$ monotonic $\uparrow$ if $t>0$ and monotonic $\downarrow$ if $t<0$.

Proof. Let $\rho_{1}=\rho(x, s, t), \tau_{1}=\tau(x, s, t)$. Then

$$
\rho(x, s+\delta, t)=\rho_{1}+\rho\left(h_{s} x, \delta, \tau_{1}\right) .
$$

This is because $\rho(x, s+\delta, t)$ may be characterized by the property that $g_{\rho(x, s+\delta, t)}$ takes some point on the local expanding horocycle curve through $k_{t}(x)$ to some point on the local contracting horocycle curve through $h_{s+\delta} x$. Now, lemma 2.4 tells us that

$$
\rho\left(h_{s} x, \delta, \tau_{1}\right)=(1 \pm \varepsilon) a\left(h_{s} x\right) \delta \tau_{1}
$$

and $\tau_{1}$ is non-zero, having the same sign as $t$.

Obviously one may show a similar fact for $\rho$ as a function of $s$ for fixed $t$.

\section{The uniform parametrization}

The flows $h$ and $k$ may be represented in such a way that they satisfy commutation relations with $g$, as in the case of the geodesic flow on $U(S)$, where $S$ has constant curvature. This parametrization is due to Margulis [Ma] and Marcus [M1]. Specifically: there are continuous functions $m$ and $n: M \times \mathbb{R} \rightarrow \mathbb{R}$, satisfying:

(1) $m(x, \cdot)$ and $n(x, \cdot)$ are increasing homeomorphisms of $\mathbb{R}$ sending 0 to 0 .

(2) $h_{m(\cdot, s)}$ and $k_{n(\cdot, t)}$ give flows $\bar{h}_{s}$ and $\bar{k}_{t}$ satisfying

$$
g_{r} \bar{h}_{s} g_{-r}=\bar{h}_{\alpha r_{s}}, \quad g_{r} \bar{k}_{t} g_{-r}=\bar{k}_{\alpha^{-r} t} .
$$

It is easy to see that $m$ and $n$ are unique up to positive multiplicative constants.

(3.1) Definition. We call these uniform parametrizations.

$\bar{h}$ and $\bar{k}$ are uniquely ergodic (as was shown by Marcus [M1]), sharing the same invariant probability measure $\mu$, which may also be characterized as the g-invariant probability measure of maximal entropy. This measure $\mu$ will always be singular, except in the 'classical' case.

(3.2) Definition. We say $g$ is normalized if the $\alpha$ arising in the commutation relation is $e$. 
$g$ may always be normalized by a constant scaling of its time parameter. In the geodesic flow case, this may be achieved by a constant scaling of the metric. Hereafter we will always assume that $g$ is so normalized.

(3.3) Definition. (i) $\bar{\rho}(x, s, t)=\rho(x, m(x, s), n(x, t))$;

(ii) $\bar{\tau}(x, s, t)=\tau(x, m(x, s), n(x, t))$; and

(iii) $\bar{\sigma}(x, s, t)=\sigma(x, m(x, s), n(x, t))$;

for small $s$ and $t$.

Notice that $\bar{\rho}\left(x, e^{r} s, e^{-r} t\right)=\bar{\rho}\left(g_{-r} x, s, t\right)$ if $e^{r} s$ and $e^{-r} t$ are also small. Thus $\bar{\rho}(x, s, t)$ may be defined whenever the product st is sufficiently small, by choosing $r$ so that $e^{r} s$ and $e^{-r} t$ are separately small, and defining $\bar{\rho}(x, s, t)=\bar{\rho}\left(g_{r} x, e^{r} s, e^{-r} t\right)$. No inconsistency is possible, because of the earlier sentence. Similar considerations hold for $\bar{\sigma}$ and $\bar{\tau}$. The equation $g_{\bar{\rho}} \bar{k}_{\bar{\tau}} \bar{h}_{s}=\bar{h}_{\bar{\sigma}} \bar{k}_{t}$ holds, as in the discussion before lemma 2.3; the picture there gets stretched to produce a distorted picture elsewhere. $\bar{\rho}$ shares the monotonicity properties of $\rho$. Notice also the 'scaling' properties of $\bar{\sigma}$ and $\bar{\tau}$ :

$$
\bar{\sigma}\left(x, e^{r} s, e^{-r} t\right)=e^{r} \tilde{\sigma}\left(g_{-r} x, s, t\right)
$$

while

$$
\bar{\tau}\left(x, e^{r} s, e^{-r} t\right)=e^{-r} \bar{\tau}\left(g_{-r} x, s, t\right) .
$$

(3.4) LemMa. For sufficiently small st,

$$
\frac{\partial \vec{\sigma}}{\partial s}(x, s, t)=e^{\bar{\rho}(x, s, t)} .
$$

Equivalently:

$$
\bar{\sigma}(x, s, t)-s=\int_{0}^{s}\left(e^{\bar{\rho}}\left(x, s^{\prime}, t\right)-1\right) d s^{\prime} .
$$

Proof. The points $g_{\bar{\rho}} \bar{h}_{s} x$ and $\bar{h}_{\bar{\sigma}} \bar{k}_{t} x$ are connected by a contracting curve of uniform length $e^{-\bar{\rho}} \bar{\tau}$, because of the definition of the quantities and the commutation relation between $\bar{k}$ and $g$. Here $\bar{\rho}, \bar{\sigma}$ and $\bar{\tau}$ are evaluated at $(x, s, t)$. When $g_{r}$ is applied to both points, they move together, and this occurs uniformly in $s$ on any finite $s$-interval, provided $t$ is fixed and $s t$ remains small. Let $s_{1}=s_{0}+e^{-r}$, and $\bar{\rho}_{i}, \bar{\sigma}_{i}, \bar{\tau}_{i}$ correspond to the points $\left(x, s_{i}, t\right)$. Now, by using $g_{\bar{\rho}_{0}} \bar{h}_{s_{0}} x$ as the starting point, $\bar{\sigma}_{1}-\bar{\sigma}_{0}$ may be written as

$$
\tilde{\sigma}\left(g_{\bar{\rho}_{0}} \bar{h}_{s_{0}} x, e^{\bar{\rho}_{0}-r}, e^{-\bar{\rho}_{0}} \bar{\tau}_{0}\right)=e^{-r} \bar{\sigma}\left(g_{r+\bar{\rho}_{0}} \bar{h}_{s_{0}} x, e^{\bar{\rho}_{0}}, e^{-\bar{\rho}_{0}-r} \bar{\tau}_{0}\right)
$$

Then

$$
\frac{\bar{\sigma}_{1}-\bar{\sigma}_{0}}{s_{1}-s_{0}}=\bar{\sigma}\left(g_{r+\bar{\rho}_{0}} \bar{h}_{s_{0}} x, e^{\bar{\rho}_{0}}, e^{-\bar{\rho}_{0}-r} \bar{\tau}_{0}\right)
$$

$\bar{\rho}_{0}$ and $\bar{\tau}_{0}$ stay fixed as $r$ varies, so $\bar{\sigma}\left(\cdot, e^{\bar{\rho}_{0}}, e^{-\bar{\rho}_{0}-r} \bar{\tau}_{0}\right) \rightarrow e^{\bar{\rho}_{0}}$ uniformly as $r \rightarrow \infty$. Thus $\left(\bar{\sigma}_{1}-\bar{\sigma}_{0}\right) /\left(s_{1}-s_{0}\right) \rightarrow e^{\bar{\rho}_{0}}$. 


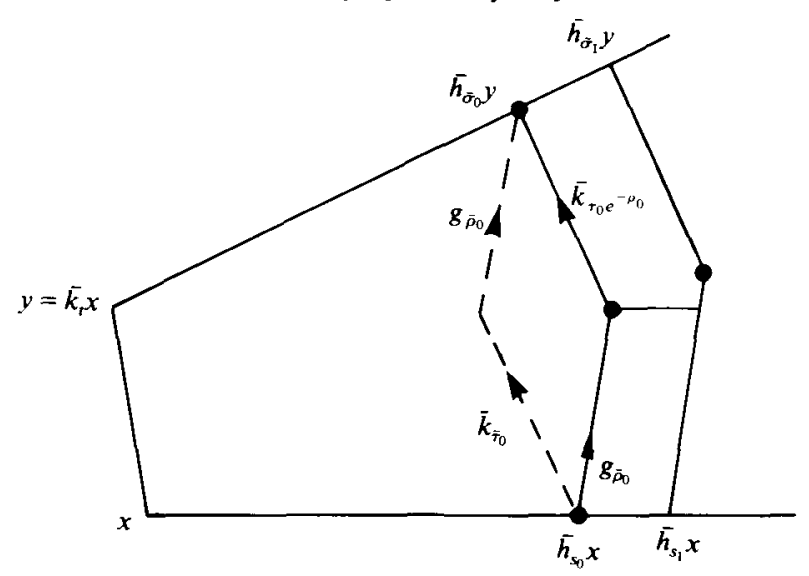

FIGURE 3

(3.5) COROllary. For sufficiently small $s t, \bar{\sigma}(x, s, t)-s$ is monotonic in $s$.

(3.6) Corollary. For sufficiently small st, $\bar{\sigma}(x, s, t)-s$ differs from $\int_{0}^{s} \bar{\rho}\left(x, s^{\prime}, t\right) d s^{\prime}$ by a factor which is uniformly close to 1 .

Proof. $e^{\bar{\rho}\left(x, s^{\prime}, t\right)}-1-\bar{\rho}\left(x, s^{\prime} t\right)$ is small compared with $\bar{\rho}\left(x, s^{\prime}, t\right)$, uniformly in $x, s^{\prime}, t\left(0 \leq s^{\prime} \leq s\right)$, provided $|s t|$ stays small.

Of course, $\bar{h}$ and $\bar{k}$ play more or less symmetric roles; for example, $\partial \bar{\tau} / \partial t=e^{-\bar{\rho}}$; this will be used later.

$\bar{\rho}$ has certain uniformity properties, reflected in the following lemma.

(3.7) LEMMA. There are positive constants $\gamma$ (depending on $\varepsilon$ ) and $\bar{\gamma}<\frac{1}{2}$ such that for all $x, s, t$, provided only that st is sufficiently small,

(a) $|(\bar{\sigma}(x, s, t)-s)-(\bar{\sigma}(x,(1-\gamma) s, t)-(1-\gamma) s)| \leq \varepsilon|\bar{\sigma}(x, s, t)-s|$

(b) $|\bar{\sigma}(x, s / 2, t)-s / 2| \leq\left(\frac{1}{2}-\bar{\gamma}\right)|\bar{\sigma}(x, s, t)-s|$.

Proof. $\bar{\sigma}(x, s, t)-s=\int_{0}^{s}\left(e^{\bar{\rho}\left(x, s^{\prime}, t\right)}-1\right) d s^{\prime}$, so because of (3.5) and (3.6) it suffices to show

(a') $\left|\int_{(1-\gamma) s}^{s} \bar{\rho}\left(x, s^{\prime}, t\right) d s^{\prime}\right| \leq \varepsilon\left|\int_{0}^{s} \bar{\rho}\left(x, s^{\prime}, t\right) d s^{\prime}\right|$

and

(b') $\left|\int_{0}^{s / 2} \bar{\rho}\left(x, s^{\prime}, t\right) d s^{\prime}\right| \leq\left(\frac{1}{2}-\bar{\gamma}\right)\left|\int_{0}^{s} \bar{\rho}\left(x, s^{\prime}, t\right) d s^{\prime}\right|$.

Let $\alpha$ be so small that $|s|,|t|<\alpha$ implies

$$
\frac{\rho(x, m(x, s), n(x, t))}{m(x, s) n(x, t)}=\left(1 \pm \frac{1}{2}\right) a(x)
$$

via proposition 2.7 . To see $\left(a^{\prime}\right)$ :

$$
\frac{\int_{(1-\gamma) s}^{s} \bar{\rho}\left(x, s^{\prime}, t\right) d s^{\prime}}{\int_{0}^{s} \bar{\rho}\left(x, s^{\prime}, t\right) d s^{\prime}} \leq \frac{\int_{(1-\gamma) s}^{s} \bar{\rho}\left(x, s^{\prime}, t\right) d t}{\int_{\frac{1}{2} s}^{s} \bar{\rho}\left(x, s^{\prime}, t\right) d s^{\prime}} \leq \frac{\gamma \bar{\rho}(x, s, t)}{\frac{1}{2} \bar{\rho}\left(x, \frac{1}{2} s, t\right)},
$$


by monotonicity of $\bar{\rho}$ in $s^{\prime}$. Setting $y=g_{\log (\alpha / s)} x$, this equals

$$
\frac{\gamma \bar{\rho}(y, \alpha, s t / \alpha)}{\frac{1}{2} \bar{\rho}(y, \alpha / 2, s t / \alpha)} \leq \frac{\gamma\left(1+\frac{1}{2}\right) a(y) m(y, \alpha) n(y, s t / \alpha)}{\frac{1}{2}\left(1-\frac{1}{2}\right) a(y) m(y, \alpha / 2) n(y, s / \alpha)}=6 \gamma \frac{m(y, \alpha)}{m(y, \alpha / 2)}
$$

But $m(\cdot, \alpha) / m(\cdot, \alpha / 2)$ is bounded on $M$, so $\gamma$ may be chosen to make the right-hand side $<\varepsilon$. This proves $\left(a^{\prime}\right)$. To see $\left(b^{\prime}\right)$ :

$$
\frac{\int_{0}^{s / 2} \bar{\rho}\left(x, s^{\prime}, t\right) d s^{\prime}}{\int_{s / 2}^{s} \bar{\rho}\left(x, s^{\prime}, t\right) d s^{\prime}}=\frac{\int_{0}^{\delta s}+\int_{\delta s}^{s / 2} \bar{\rho}\left(x, s^{\prime}, t\right) d s^{\prime}}{\int_{(1-\delta) s}^{s}+\int_{s / 2}^{(1-\delta) s} \bar{\rho}\left(x, s^{\prime}, t\right) d s^{\prime}} .
$$

By monotonicity, $\int_{\delta s}^{s / 2} \bar{\rho}\left(x, s^{\prime}, t\right) d s^{\prime} \leq \int_{s / 2}^{(1-\delta) s} \bar{\rho}\left(x, s^{\prime}, t\right) d s^{\prime}$, so dividing the numerator and denominator by $\int_{s / 2}^{(1-\delta) s} \bar{\rho}\left(x, s^{\prime}, t\right) d s^{\prime}$ and using this fact, our expression is no greater than

$$
\frac{\int_{0}^{\delta s} / \int_{s / 2}^{(1-\delta) s}+1}{\int_{(1-\delta) s}^{s} / \int_{s / 2}^{(1-\delta) s}+1} \leq \frac{\int_{0}^{\delta s} / \int_{s / 2}^{(1-\delta) s}+1}{\delta /\left(\frac{1}{2}-\delta\right)+1}
$$

using monotonicity at the last step. Now,

$$
\frac{\int_{0}^{\delta s} \bar{\rho}\left(x, s^{\prime}, t\right) d s^{\prime}}{\int_{s / 2}^{(1-\delta) s} \bar{\rho}\left(x, s^{\prime}, t\right) d s^{\prime}} \leq \frac{\delta \bar{\rho}(x, \delta s, t)}{\left(\frac{1}{2}-\delta\right) \bar{\rho}(x, s / 2, t)},
$$

again by monotonicity. As before, we 'scale' to get this equal to

$$
\frac{\delta}{\left(\frac{1}{2}-\delta\right)} \frac{\bar{\rho}(y, \delta \alpha, s t / \alpha)}{\bar{\rho}(y, \alpha / 2, s t / \alpha)} \leq \frac{\delta}{\left(\frac{1}{2}-\delta\right)} \frac{\left(1+\frac{1}{2}\right)}{\left(1-\frac{1}{2}\right)} \frac{m(y, \delta \alpha)}{m(y, \alpha / 2)}
$$

But $m(\cdot, \delta \alpha) \rightarrow 0$ uniformly as $\delta \rightarrow 0$, so for sufficiently small $\delta$, this is less than or equal to $\left(\delta /\left(\frac{1}{2}-\delta\right)\right) \cdot \frac{1}{2}$. Thus the entire expression is less than or equal to

From this, ( $\left.b^{\prime}\right)$ follows immediately.

$$
\frac{\frac{\delta}{\left(\frac{1}{2}-\delta\right)} \cdot \frac{1}{2}+1}{\frac{\delta}{\left(\frac{1}{2}-\delta\right)}+1}<1
$$

(3.8) LemmA. Given $\varepsilon>0$, $\alpha$ may be chosen so small that if $y=g_{u} \bar{h}_{v} \bar{k}_{w} x$ with $|u|,|v|$, $|w|<\alpha$, and $|s w|<\alpha^{2} / 2$, then $d\left(\bar{h}_{s} x, \bar{h}_{e}^{u} \bar{\sigma}(x, s, w) y\right)<\varepsilon$.

Proof.

$$
\begin{aligned}
\bar{h}_{e^{u} \bar{\sigma}(x, s, w)} y & =\bar{h}_{e^{u} \bar{\sigma}(x, s, w)} g_{u} \bar{h}_{v} \bar{k}_{w} x=g_{u} \bar{h}_{v} \bar{h}_{\bar{\sigma}(x, s, w)} \bar{k}_{w} x \\
& =g_{u} \bar{h}_{v} \bar{k}_{\bar{\tau}(x, s, w)} g_{\bar{\rho}(x, s, w)} \bar{h}_{s} x .
\end{aligned}
$$

Now: $|u|$ and $|v|$ are small by assumption;

$$
|\bar{\rho}(x, s, w)|=\left|\bar{\rho}\left(g_{\log w / \alpha} x, s w / \alpha, \alpha\right)\right| \leq \sup |\bar{\rho}(\cdot, \alpha / 2, \alpha)|
$$


by monotonicity, so $|\bar{\rho}(x, s, w)|$ is small. Finally, $\bar{\tau}(x, s, w) / w \approx 1$, so $|\bar{\tau}(x, s, w)|$ is small. Thus, $g_{u} \bar{h}_{v} \bar{k}_{\bar{\tau}(x, s, w)} g_{\bar{\rho}(x, s, w)} \bar{h}_{s} x$ is close to $\bar{h}_{s} x$.

(3.9) LEMMA (Ratner's $\mathscr{H}_{p}$ property). Given $\varepsilon_{0}>0, D>0$, and $N_{0}>0$, then there exists $\delta>0$ and $\beta>0$ so that if $d(x, y)<\delta$ and $x, y$ are not on the same $\bar{h}$ orbit, then for some $N>N_{0}$ either $\bar{h}_{s} x$ is within $\varepsilon_{0}$ of $\bar{h}_{s-D} y$ for all $s$ in $[(1-\beta) N, N]$ or $\bar{h}_{s} x$ is within $\varepsilon_{0}$ of $\bar{h}_{s+D} y$ for all $s$ in $[(1-\beta) N, N]$.

Proof. Write $y=g_{u} \bar{h}_{v} \bar{k}_{w} x$ in local coordinates. $\delta$ small will make $|u|,|v|,|w|$ small.

First suppose $w=0$. Then $u \neq 0$, and $y=g_{u} \bar{h}_{v} x$, and $\bar{h}_{e} u_{s} y=g_{u} \bar{h}_{v} \bar{h}_{s} x$. If $\delta$ is small enough then this is within $\varepsilon_{0} / 2$ of $\overline{h_{s}} x$. Now choose $N$ so $\left|1-e^{u}\right| N=D$; if $\delta$ is small then $N>N_{0}$. If $(1-\beta) N \leq s \leq N$, then $\left|s-e^{u} s\right|$ will be between $(1-\beta) D$ and $D$. If $\beta$ is a sufficiently small pre-assigned number, then $\bar{h}_{e^{u}} y$ will be within $\varepsilon_{0} / 2$ of $\bar{h}_{s \pm D} y$, and we are done.

If $w \neq 0$, let $\varepsilon=\varepsilon_{0} / 2$ and take $\alpha$ from the last lemma. Then $\left|\bar{h}_{s} x-\bar{h}_{e^{u} \bar{\sigma}(x, s, w)} y\right| \leq \varepsilon_{0} / 2$ provided $|u|,|v|,|w|<\alpha$ and $|s w|<\alpha^{2} / 2$. We try to choose $N$ with $|N w|<\alpha^{2} / 2$ and $\left|e^{u} \bar{\sigma}(x, N, w)-N\right|=D$. Let $r(s)=\left|e^{u} \bar{\sigma}(x, s, w)-s\right|, A(s)=\bar{\sigma}(x, s, w)-s$. Then $r(s)=$ $\left|e^{u} A(s)+\left(e^{u}-1\right) s\right|$. By choosing $\delta$ small we make $|w|$ small, and setting $L=\alpha^{2} / 2 w$, a scaling argument tells us that $A(L)$ can be made as large as desired. If $|r(L)|<D$ then $A(L)$ and $\left(e^{u}-1\right) L$ must have opposite signs. Then

$$
\begin{aligned}
r\left(\frac{L}{2}\right) & \geq\left|\left(e^{u}-1\right) \frac{L}{2}\right|-\left|e^{u} A\left(\frac{L}{2}\right)\right| \\
& \geq\left|\left(e^{u}-1\right) \frac{L}{2}\right|-\left(\frac{1}{2}-\bar{\gamma}\right)\left|e^{u} A(L)\right|
\end{aligned}
$$

(from lemma $3.7(b)$ )

$$
\geq \bar{\gamma}\left|e^{u} A(L)\right|-\frac{1}{2} r(L) \geq \bar{\gamma}\left|e^{u} A(L)\right|-D / 2 .
$$

So in any case there is some smallest number $N$ with $r(N)=D$; and $0 \leq N \leq \alpha^{2} / 2 w$. Furthermore, smallness of $w$ forces $N$ to be larger than $N_{0}$.

Now: $\quad D \geq r(N / 2) \geq \bar{\gamma}\left|e^{u} A(N)\right|-(D / 2)$, so $\left|e^{u} A(N)\right| \leq(3 D / 2) \bar{\gamma}$, and $\left|\left(e^{u}-1\right) N\right| \leq(1+3 / 2 \bar{\gamma}) D$. So if $\beta$ is sufficiently small and $s$ is in $[(1-\beta) N, N]$ then by lemma $3.7(\mathrm{a}), A(s)$ will be close to $A(N)$, and obviously also $\left(e^{u}-1\right) s$ to $\left(e^{u}-1\right) N$. So

$$
e^{u} \bar{\sigma}(x, s, w)=s+e^{u} A(s)+\left(e^{u}-1\right) s,
$$

which is close to $s \pm D$, and we are done.

Next, we generalize the 'Basic Lemma' of [R1].

(3.10) Corollary. Given $N_{0}>0$, there is some $\beta>0$ and some $\delta>0$ such that if $x$ and $y$ are closer than $\delta$, and not on the same expanding curve, then for some $N>N_{0}$, $\bar{h}_{s} x$ and $\bar{h}_{s} y$ will be no closer than $\beta$, for all $s$ between $(1-\beta) N$ and $N$.

Proof. Choose $D$ so that $\bar{h}_{s} x$ and $\bar{h}_{s \pm D} x$ can never be closer than some fixed distance. But $\bar{h}_{s \pm D} x$ will be very close to $\bar{h}_{s} y$ for $s$ in the appropriate range; thus $\bar{h}_{s} x$ cannot be very close to $\bar{h}_{s} y$. 
(3.11) Corollary. Suppose $(g, M)$ and $\left(g^{\prime}, M^{\prime}\right)$ are good, normalized Anosov flows, and $\phi:\left(\bar{h}_{1}, \mu\right) \rightarrow\left(\bar{h}_{1}^{\prime}, \mu^{\prime}\right)$ is a conjugacy between their respective uniformly parametrized expanding flows at time 1 . Then there is a real number $s_{0}$, a full measure set $M_{0} \subset M$, invariant under both $g$ and $\bar{h}$, and a bimeasurable map $\psi: M_{0} \rightarrow M$ which carries $\bar{h}$ to $\bar{h}^{\prime}$ and $g$ to $g^{\prime}$, and such that $\phi=\bar{h}_{s_{0}}^{\prime} \psi$ a.e.

Proof. This may be shown exactly as in [R1]. For expository reasons, we include a very brief sketch of the argument. By Lusin's theorem, there is a set $X$ of measure nearly 1 on which $\phi$ is uniformly continuous. Choose $x$ and $r$ so both $x$ and $y=g_{r} x$ lie in $X$. If $r$ is small, then $x^{\prime}=\phi x$ will be close to $y^{\prime}=\phi y$. Now $g_{r} \bar{h}_{s} x=\bar{h}_{e^{\prime} s} y$, so $\bar{h}_{s} x$ and $\bar{h}_{e^{\prime} s} y$ are close, uniformly in $s$, provided $r$ is small enough. Then if both $\bar{h}_{s} x$ and $\bar{h}_{e^{r} s} y$ happen to be in $X$, as will indeed be the case for a large proportion of $s>0$ because of ergodicity of $\bar{h}$, then $\bar{h}_{s}^{\prime} x^{\prime}=\phi\left(\bar{h}_{s} x\right)$ will be close to $\bar{h}_{e^{\prime} s}^{\prime} y^{\prime}=\phi\left(\bar{h}_{e^{\prime}} y\right)$, uniformly in $s$. But also $\bar{h}_{s}^{\prime} x^{\prime}$ is close to $\bar{g}_{r}^{\prime} \bar{h}_{s}^{\prime} x^{\prime}=\bar{h}_{e^{r} s} g_{r}^{\prime} x^{\prime}$, uniformly in $s$ (see figure 4).

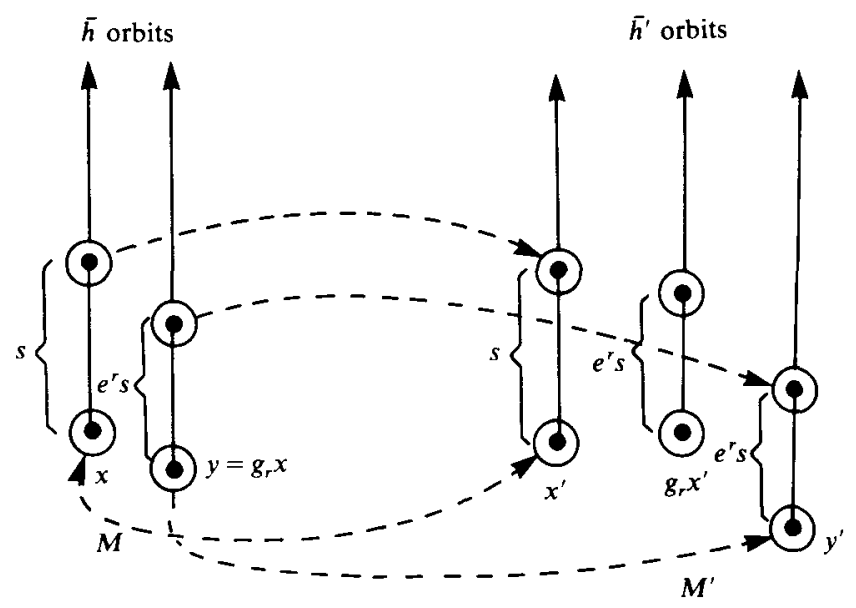

FIGURE 4

Then by lemma 3.9, $y^{\prime}$ must be of the form $\bar{h}_{s_{0}}^{\prime} g_{r}^{\prime} x^{\prime}$, where $s_{0}$ depends on $x$ and $r$. It is then not difficult to verify that this works for all $r$ and a.e. $x$, and that $s_{0}$ is in fact a constant.

\section{Conjugacy of the contracting flows}

(4.1) Proposition. Let $(g, M)$ and $\left(g^{\prime}, M^{\prime}\right)$ be good, normalized Anosov flows, and let $\psi$ be a measurable conjugacy from $(g, \bar{h}, \mu)$ to $\left(g^{\prime}, \bar{h}^{\prime}, \mu^{\prime}\right)$. Assume that $\{\mathrm{g}, \bar{h}, \bar{k}\}$ and $\left\{g^{\prime}, \bar{h}^{\prime}, \bar{k}^{\prime}\right\}$ are properly oriented. Then there is some $C>0$ for which $\psi$ carries $\left(\bar{k}_{t}, \mu\right)$ to $\left(\bar{k}_{C t}^{\prime}, \mu^{\prime}\right)$ for all $t$.

Proof. Choose small, positive $\theta$. By Lusin's theorem, there is some $X \subset M, \mu(M)>$ $1-(\theta / 100)$, with $\psi$ uniformly continuous on $X$ and $\psi^{-1}$ on $X^{\prime}=\psi(X)$. By ergodicity of $\bar{h}$, there exists $Y \subset M, \mu(Y)>1-(\theta / 100)$, and a positive number $I$ so that if $x \in Y$ and $N>I$ then $\left|\left\{s \in[0, N]: \bar{h}_{s} x \in X\right\}\right|>(1-\theta / 50) N$. Let $W=X \cap Y$. The 
image $Y^{\prime}=\psi(Y)$ has the analogous property for $\bar{h}^{\prime}$. Let $W^{\prime}=X^{\prime} \cap Y^{\prime}$. Note that $W^{\prime}=\psi(W)$, and that $\mu(W)>1-(\theta / 50)$.

The argument now proceeds in several steps: (4.2)-(4.6).

(4.2) LEMMA. There is some $\delta>0$ so that if $0<|t|<\delta$, and $x$ and $y$ (where $y=\bar{k}_{t} x$ ) are both in $W$, and we write $x^{\prime}=\psi(x)$ and $y^{\prime}=\psi(y)$, and write $y^{\prime}$ as $g_{u}^{\prime} \bar{h}_{v}^{\prime} \bar{k}_{w}^{\prime} x^{\prime}$ with $u, v, w$ small (as may be done when $\delta$ is small enough), then $w / t>0$.

Proof. There is no loss of generality in assuming $t>0$, since the roles of $x$ and $y$ could be reversed. Fix $D>0$ as in the proof of lemma 3.11. Choose $\beta>0$ so that if $\bar{h}_{s_{1}}^{\prime} x$ and $\bar{h}_{s_{2}}^{\prime} x^{\prime}$ are closer than $\beta$, then $\left|s_{1}-s_{2}\right|<D / 2$. Choose $\gamma$ so that if $x_{1}$ and $x_{2}$ are in $X$ and within $\gamma$ of each other then $\psi x_{1}$ and $\psi x_{2}$ are within $\beta / 3$ of each other. Let $N=\left(\alpha^{2} / 2\right)(1 / t v|w|)$. If $\alpha$ is small enough, and $0<s<N$, and $\delta$ is small enough, and $0<t<\delta$, then:

(a) $\bar{h}_{s} x$ and $\bar{h}_{\bar{\sigma}(x, s, t)} y$ are within $\gamma$ of each other;

(b) if $\bar{h}_{s} x$ and $\bar{h}_{\bar{\sigma}(x, s, t)} y$ are in $X$, then $\bar{h}_{s}^{\prime} x^{\prime}$ and $\bar{h}_{\bar{\sigma}(x, s, t)}^{\prime} y^{\prime}$ are within $\beta / 3$ of each other;

(c) writing $z^{\prime}$ for $\bar{k}_{w}^{\prime} x, \bar{h}_{s}^{\prime} x^{\prime}$ and $\bar{h}_{\bar{\sigma}^{\prime}\left(x^{\prime}, s, w\right)}^{\prime} z^{\prime}$ are within $\beta / 3$ of each other;

(d) $\bar{h}_{\bar{\sigma}^{\prime}\left(x^{\prime}, s, w\right)}^{\prime} z^{\prime}$ and $\bar{h}_{e^{u} \bar{\sigma}^{\prime}\left(x^{\prime}, s, w\right)} y^{\prime}$ are within $\beta / 3$ of each other; and therefore

(e) if $\bar{h}_{s} x$ and $\bar{h}_{\bar{\sigma}(x, s, t)} y$ are in $X$ then $\bar{h}_{\bar{\sigma}^{\prime}(x, s, t)}^{\prime} y^{\prime}$ and $\bar{h}_{e^{u} \bar{\sigma}^{\prime}\left(x^{\prime}, s, w\right)}^{\prime} y$ are within $\beta$ of each other.

But we shall show that if $w<0$ and $\delta$ is small enough then there is some $s$ with $\bar{h}_{s} x$ and $\bar{h}_{\bar{\sigma}(x, s, t)} y$ in $X$ and $\left|\bar{\sigma}(x, s, t)-e^{u} \bar{\sigma}^{\prime}\left(x^{\prime}, s, w\right)\right|$ close to $D$. This will provide a contradiction.

The argument has similarities to the proof of lemma 3.10. Write $q(s)$ for $\mid \bar{\sigma}(x, s, t)-$ $e^{u} \bar{\sigma}^{\prime}\left(x^{\prime}, s, w\right) \mid, A(s)$ for $\bar{\sigma}(x, s, t)-s, A^{\prime}(s)$ for $\bar{\sigma}^{\prime}\left(x^{\prime}, s, w\right)-s$. Notice that $A(s)>0$ and $A^{\prime}(s)<0$, since we are assuming $w<0$. So

$$
q(s)=\left|A(s)-e^{u} A^{\prime}(s)-\left(e^{u}-1\right) s\right| .
$$

Suppose $q(N)<D$. Now, if $N=\alpha^{2} / 2 t$, then $A(N)$ is large, by scaling. If $N=$ $\alpha^{2} / 2|w|$, then $-e^{u} A^{\prime}(N)$ is large, for the same reason. In any case, $A(N)-e^{u} A^{\prime}(N)$ is large; so also $\left(e^{u}-1\right) N$ is large, and positive, always provided $\delta$ is small enough.

Now by $(3.7(\mathrm{~b}))$, there is some $\bar{\gamma}, 0<\bar{\gamma}<\frac{1}{2}$, (chosen to work in both $M$ and $M^{\prime}$ ) for which $A(N / 2)<\left(\frac{1}{2}-\gamma^{\prime}\right) A(N)$ and $A^{\prime}(N / 2)<\left(\frac{1}{2}-\bar{\gamma}\right) A^{\prime}(N)$, so

and

$$
A(N / 2)-e^{u} A^{\prime}(N / 2)<\left(\frac{1}{2}-\bar{\gamma}\right)\left(A(N)-e^{u} A^{\prime}(N)\right) \text {, }
$$

$$
\begin{aligned}
q(N / 2) & \geq\left(e^{u}-1\right) N / 2-\left(A(N / 2)-e^{u} A^{\prime}(N / 2)\right) \\
& >\bar{\gamma}\left(e^{u}-1\right) N-\left(\frac{1}{2}-\bar{\gamma}\right)\left(A(N)-e^{u} A^{\prime}(N)-\left(e^{u}-1\right) N\right) \\
& >\bar{\gamma}\left(e^{u}-1\right) N-\left(\frac{1}{2}-\bar{\gamma}\right) D .
\end{aligned}
$$

But if $\delta$ is small enough then $\left(e^{u}-1\right) N$ gets large, making this $>D$. So in any case, $\delta$ small enough forces $q(s) \geq D$ somewhere. Let $L$ be the smallest $s>0$ with $q(s)=D$.

Now we see that $\left(e^{u}-1\right) L, A(L)$, and $A^{\prime}(L)$ are all bounded by a fixed constant multiple of $D$. Arguing as before: $D>q(L / 2)>\bar{\gamma}\left(e^{u}-1\right) L-\left(\frac{1}{2}-\bar{\gamma}\right) D$, so

$$
\left(e^{u}-1\right) L<\frac{\frac{3}{2}-\bar{\gamma}}{\bar{\gamma}} D
$$


and a similar bound follows for $A(L)-e^{r} A^{\prime}(L)$ and hence for $A(L)$ and $-e^{r} A^{\prime}(L)$ separately. But also, making $\delta$ small makes $\left(e^{u}-1\right) s, A(s)$, and $e^{u} A^{\prime}(s)$ small for any fixed $s$, so $L$ can be made large by making $\delta$ small.

Let $S=\left\{s \in[0, L]: \bar{h}_{s} x \in X\right\}$, and $T=\left\{s \in[0, \bar{\sigma}(x, L, t)]: \bar{h}_{s} y \in X\right\}$. If $L$ is large enough then $|s|>(1-(\theta / 50)) L$; and since $\bar{\sigma}(x, L, t)$ will also be large, and since the derivative of the map $s \mapsto \sigma(x, s, t)$ is near 1 , the inverse image of $T$ in $[0, L]$ has measure $>(1-(\theta / 25)) L$. So there is some $s$ in $[(1-\theta) L, L]$ with $\bar{h}_{s} x$ and $\bar{h}_{\bar{\sigma}(x, s, t)} y$ in $X$. If $\theta$ is small enough, then by corollary 3.7(a) the terms $\left(e^{u}-1\right) s$ and $A(s)-$ $e^{u} A^{\prime}(s)$ will differ little from $\left(e^{u}-1\right)$ and $A(L)-e^{u} A^{\prime}(L)$. So $q(s)$ differs little from $q(L)=D$, proving the lemma.

Next we need some measure-theoretic discussion. Let us choose our basic neighbourhoods more carefully. A local contracting (LC) curve is a curve of the form $t \mapsto \bar{k}_{t} x$, for small values of $t$. A local weakly expanding (LWE) surface is a surface of the form $(r, s) \mapsto g_{r} \bar{h}_{s} x$, for small values of $r$ and $s$. A local cylinder will be a subset $P$ of $M$ which is obtained by taking two nearby LWE surfaces $D_{0}$ and $D_{1}$ such that every point of $D_{0}$ is connected to a point of $D_{1}$ by an LC curve. Dually, $P$ is the union of all the points on the LC curves from $D_{0}$ to $D_{1}$. Thus $P$ is a union of LWE surfaces, and the Poincaré map along LC curves is 1-1 onto between any two of these LWE surfaces. Similarly, $P$ is a union of LC arcs, and the Poincaré map along LWE surfaces is 1-1 onto between any two of these LC curves.

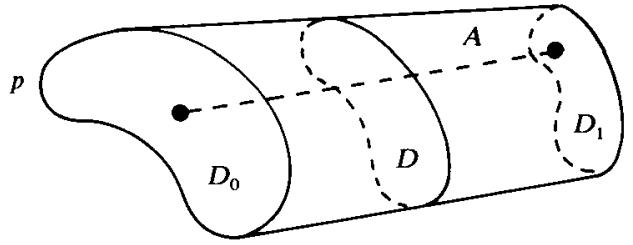

FIGURE 5

In figure 5, $D_{0}, D, D_{1}$ are LWE surfaces in $P$, called 'disks', while $A$ is an LC curve, called a 'segment'.

Now we introduce coordinates: in a local cylinder $P$ containing $x$, we send the point where the segment through $g_{r} \bar{h}_{s} x$ meets the disk through $\overline{k_{r}} x$, to the triple $(r, s, t)$. Call this the standard coordinate system with $x$ as origin. Notice that then the measure $\mu$ is, infinitesimally, a fixed multiple of $d r d s d t$; more precisely, we state without proof the following, which may be deduced from the descriptions of $\mu$ given, for example, in [B-M] and [M1].

(4.3) Proposition. (a) The conditional measure on LWE surfaces is proportional to hyperbolic measure on the Poincaré upper half-plane, while that on $\mathrm{LC}$ curves is proportional to the parameter length.

(b) The Poincaré map along LC curves, from LWE surface to LWE surface, preserves this conditional measure; and likewise the Poincaré map along LWE surfaces from $\mathrm{LC}$ curve to $\mathrm{LC}$ curve, preserves conditional measure.

(c) There is a $B>0$ such that for any $\varepsilon>0$, if $P$ is a sufficiently small local cylinder, and we put on $P$ the standard coordinate system at $x \in P$, then the image of $\mu \mid P$ is 
within a factor $[1 /(1+\varepsilon), 1+\varepsilon]$ of $B \times$ Lebesgue measure on the coordinate image $\tilde{P}$ of $P$.

Concerning (a): this is fairly obvious from the fact that $\mu$ is invariant under $g, \bar{h}$, and $\bar{k}$. Concerning (b): in those arguments where (b) is used, absolute continuity would actually have sufficed. Concerning (c): we let $B^{\prime}$ be the corresponding constant for $M^{\prime}$, and let $C=B / B^{\prime}$. The contracting horocycles could, of course, have been scaled so that $B=B^{\prime}=1$.

(4.4) Lemma. Given $\varepsilon_{1}>0$, there exists $\delta_{1}>0$ such that if $0<t_{1}<\delta_{1}$ then the following set $U_{t_{1}}$ has measure $>1-\varepsilon_{1}: x_{1} \in U_{t_{1}}$ if, writing $y_{1}=\bar{k}_{t_{1}} x_{1}, x_{1}^{\prime}=\psi x_{1}, y_{1}^{\prime}=\psi y_{1}$, then $y_{1}^{\prime}$ may be written as $g_{u_{1}}^{\prime} \bar{h}_{v_{1}}^{\prime} \bar{k}_{w_{1}}^{\prime} x_{1}^{\prime}$ with $\left|u_{1}\right|,\left|v_{1}\right|,\left|w_{1}\right|<\varepsilon_{1}$ and $0<w_{1} / t_{1}<C+\varepsilon_{1}$.

Proof. By the Lebesgue density theorem, almost all points of $W^{\prime}$ are Lebesgue density points with respect to conditional measure on LC curves. Let $M_{0}$ be as in corollary 3.11. Let $M_{0}^{\prime}=\psi\left(M_{0}\right)$, and let $\Lambda$ be the set of $x^{\prime}$ in $M_{0}^{\prime} \cap W^{\prime}$ for which, if $0<w<\beta_{0}$, a proportion at least $1-\theta$ of the segment $\left\{\bar{k}_{t^{\prime}} x^{\prime}: 0 \leq t^{\prime} \leq w\right\}$ lies in $M_{0}^{\prime} \cap W^{\prime}$. Since $\mu\left(W^{\prime}\right)>1-(\theta / 25), \beta_{0}$ may be chosen so small that $\mu^{\prime}(\Lambda)>1-(\theta / 20)$.

Let $D_{x}(\alpha)=\left\{g_{r} \bar{h}_{s} x:|r|,|s| \leq \alpha\right\}$ and $S_{x}(\beta)=\left\{\bar{k}_{t} x: 0 \leq t \leq \beta\right\}$. If $\alpha$ and $\beta$ are small, then there is a (unique) local cylinder $P_{x}(\alpha, \beta)$ containing $D_{x}(\alpha)$ as a disk and $S_{x}(\beta)$ as a segment. Now choose $\varepsilon>0$, and subject $\beta_{0}$ to the further condition that $P_{x}\left(\beta_{0}, \beta_{0}\right)$ always exists and satisfies proposition 4.3 for this $\varepsilon$, and analogously for the similarly defined $P_{x^{\prime}}^{\prime}\left(\beta_{0}, \beta_{0}\right)$ containing $D_{x^{\prime}}^{\prime}\left(\beta_{0}\right)$ and $S_{x^{\prime}}^{\prime}\left(\beta_{0}\right), x^{\prime}$ in $M^{\prime}$.

In a similar manner to the first condition on $\beta_{0}$, we may choose $\alpha_{0}$ so small that if $\Delta$ is the set of $x$ in $M_{0}^{\prime} \cap W^{\prime}$ for which at least $9 / 10$ of the conditional measure of $D_{x^{\prime}}^{\prime}\left(\alpha_{0}\right)$ lies in $\Lambda$, then $\mu^{\prime}(\Delta)>1-(\theta / 10), \alpha_{0}$ should also be smaller than $\beta_{0} / 2$.

Choose positive $\varepsilon_{0} \leq \beta_{0}$. We shall also want $\varepsilon_{0} / \alpha_{0}$ small; exactly how small will be seen later.

Now choose positive $\delta_{0}<\alpha_{0}$, smaller than the $\varepsilon_{1}$ of this lemma. It should be so small that all segments of $P_{x^{\prime}}\left(\alpha_{0}, \delta_{1}\right)$ are shorter than the $\delta$ which would work for lemma 4.2 using $\psi^{-1}$ instead of $\psi$. $\delta_{0}$ will also satisfy another smallness condition, which will be described later.

Next choose positive $\delta_{1}<\alpha_{0}$, and smaller than the $\delta$ of lemma 4.2, and so small that if $x$ and $\bar{k}_{t} x$ are in $W$ and $\psi\left(\bar{k}_{t} x\right)=g_{u}^{\prime} \bar{h}_{v}^{\prime} \bar{k}_{w}^{\prime} \psi(x)$ and $0<t<\delta_{1}$ then $|u|,|v|,|w|<$ $\delta_{0}$. There will be another smallness condition on $\delta_{1}$, to be described later.

Choose any $t_{1}, 0<t_{1}<\delta_{1}$. The set $V_{t_{1}}=\bar{k}_{-t_{1}}\left(M_{0} \cap W\right) \cap \psi^{-1}(\Delta)$ has measure $>1-(\theta / 25)-(\theta / 10)>1-\theta$; we claim it is contained in $U_{t_{1}}$.

For, choose any $x_{1}$ in $V_{t_{1}}$. Let $P=P_{x_{1}}\left(\alpha_{0}, t_{1}\right), D_{0}=D x_{1}\left(\alpha_{0}\right)$, its 'bottom', and $D_{1}$ the disk in $P$ through $\vec{k}_{t_{1}} x_{1}=y_{1}$, i.e. the 'top' of $P$. Likewise we set $R=P_{x_{1}}\left(\alpha_{0}+\varepsilon_{0}, t_{1}\right)$, $E_{0}$ its bottom, $E_{1}$ its top. Similarly we define $P^{\prime}=P_{x_{j}}^{\prime}\left(\alpha_{0}, w_{1}\right)$, where $x_{1}^{\prime}=\psi\left(x_{1}\right)$, and $y_{1}^{\prime}=\psi\left(y_{1}\right)=g_{u_{1}}^{\prime} \bar{h}_{v_{1}}^{\prime} \bar{k}_{w_{1}}^{\prime} x_{1}^{\prime}$; and set $D_{0}^{\prime}=$ its bottom, $D_{1}^{\prime}$ its top. Also set $R^{\prime}=$ $P_{x_{1}}^{\prime}\left(\alpha_{0}+\varepsilon_{0}, w_{1}\right), E_{0}^{\prime}$ its bottom, $E_{1}^{\prime}$ its top. Now, $\psi\left(D_{0}\right)=D_{0}^{\prime}$, but $\psi\left(D_{1}\right) \neq D_{1}^{\prime}$. However, if $\delta_{0}$ is sufficiently small, and if $x^{\prime} \in D_{0}^{\prime}, y^{\prime}=\bar{k}_{w}^{\prime} x^{\prime} \in D_{1}^{\prime}, x=\psi^{-1} x^{\prime}$, and $y=\psi^{-1} y^{\prime}$, then the point $\bar{k}_{t} x$ where the segment over $x$ meets $D_{1}$ is very close to $y$, and in the same LWE surface, so that $y$ is the image under the Poincare map from $E_{0}$ to $E_{1}$ of some point close to $x$; that is, $\psi^{-1}\left(D_{1}^{\prime}\right) \subset E_{1}$. Similarly $\delta_{1}$ may be chosen so small that $\psi\left(D_{1}\right) \subset E_{1}^{\prime}$. 


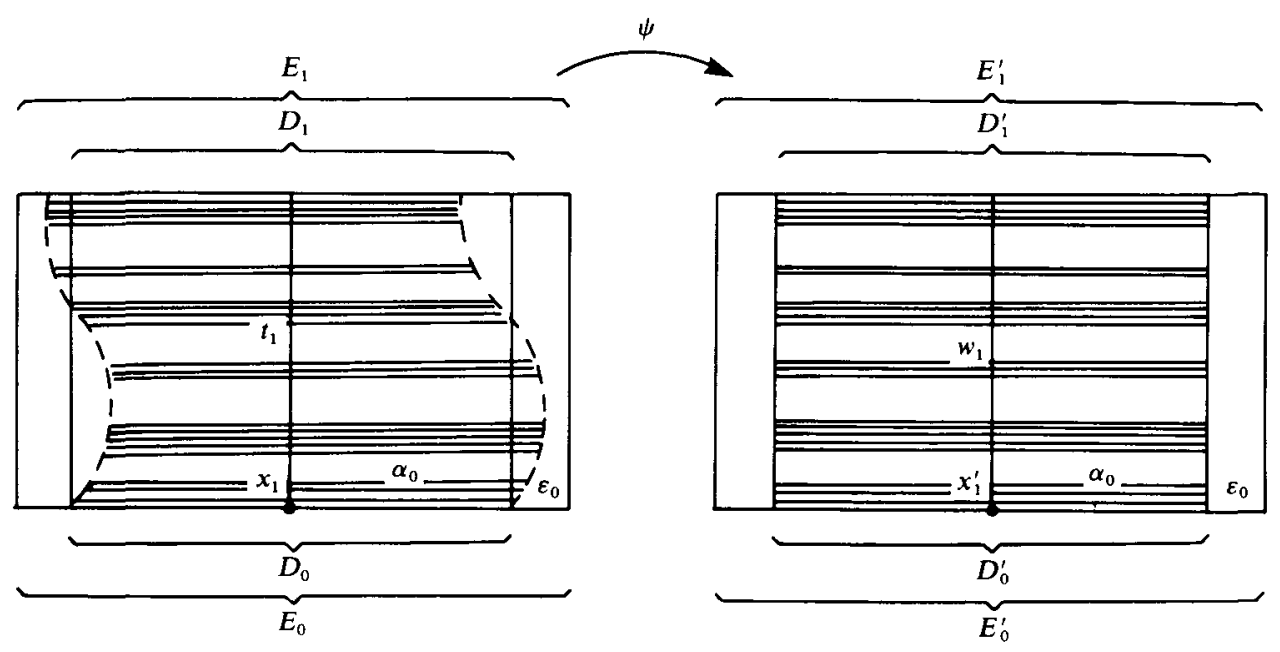

FIGURE 6. A sketch, as if $L W E$ surfaces were one-dimensional. $\psi$ carries horizontal shading on left to horizontal shading on right, sheetwise.

Let $\nu$ represent the conditional measure on $E_{1}, \nu^{\prime}$ that on $E_{1}^{\prime}$. Then

$$
\begin{aligned}
\frac{\nu^{\prime}\left(D_{1}^{\prime} \backslash \psi\left(D_{1}\right)\right)}{\nu^{\prime}\left(D^{\prime}\right)} & \leq \frac{\nu^{\prime}\left(E_{1}^{\prime} \backslash \psi\left(D_{1}\right)\right)}{\nu^{\prime}\left(D_{1}^{\prime}\right)}=\frac{\nu^{\prime}\left(E_{1}^{\prime}\right)}{\nu^{\prime}\left(D_{1}^{\prime}\right)}-\frac{\nu^{\prime}\left(\psi\left(D_{1}\right)\right)}{\nu^{\prime}\left(D_{1}^{\prime}\right)} \\
& =\frac{\nu^{\prime}\left(E_{1}^{\prime}\right)}{\nu^{\prime}\left(D_{1}^{\prime}\right)}-\frac{\nu^{\prime}\left(\psi\left(D_{1}\right)\right)}{\nu^{\prime}\left(\psi\left(E_{1}\right)\right)}=\frac{\nu^{\prime}\left(E_{1}^{\prime}\right)}{\nu^{\prime}\left(D_{1}^{\prime}\right)}-\frac{\nu\left(D_{1}\right)}{\nu\left(E_{1}\right)}
\end{aligned}
$$

If $\varepsilon_{0}$ is small enough compared to $\alpha_{0}$ then this will be $<1 / 10$. Since $\Lambda \cap D_{0}^{\prime}$ contains $9 / 10$ of the measure of $D_{0}^{\prime}$, and since the Poincaré map from $D_{0}^{\prime}$ to $D_{1}^{\prime}$ along segments is measure-preserving, there must be some point $x_{2}^{\prime}$ in $\Lambda \cap D_{0}^{\prime}$ so that the segment through $x_{2}^{\prime}$ hits $D_{1}^{\prime}$ in a point $\bar{k}_{w_{2}}^{\prime} x_{2}^{\prime}$ of $W^{\prime}, w_{2}>0$.

Write $x_{2}$ for $\psi^{-1} x_{2}^{\prime}$, and write $\psi^{-1} \bar{k}_{w_{2}}^{\prime} x_{2}^{\prime}$ as $g_{r_{2}} \bar{h}_{s_{2}} \bar{k}_{t_{2}} x_{2}$. Then $t_{2}>0$, by proposition 4.2. If $0<w<w_{2}$ and $\bar{k}_{w}^{\prime} x_{2}^{\prime} \in W^{\prime}$, and we write $\psi^{-1}\left(\bar{k}_{w}^{\prime} x_{2}^{\prime}\right)$ as $g_{r} \bar{h}_{s} \bar{k}_{t} x_{2}$, then again $0<t<t_{2}$, by proposition 4.2. If also $\bar{k}_{w}^{\prime} x_{2}^{\prime}$ is in $M_{0}^{\prime}$, then if $\delta_{0}$ was originally chosen small enough, the entire disk $D^{\prime}$ of $P^{\prime}$ through $\bar{k}_{w}^{\prime} x_{2}^{\prime}$ is mapped by $\psi^{-1}$ into the disk $E$ through $\psi^{-1}\left(\bar{k}_{w}^{\prime} x_{2}^{\prime}\right)$ in $R$ (as before with $D_{1}^{\prime}$ and $E_{1}$, although it becomes slightly more intricate). Thus, if we set $J=M_{0}^{\prime} \cap W^{\prime} \cap$ (segment through $x_{2}^{\prime}$ ), then the image of $J$ in any other segment is also mapped by $\psi^{-1}$ into $R$. Now, $J$ has $1-\theta$ of the length of the segment through $x^{\prime}$; and since the Poincaré map is measure-preserving, $1-\theta$ of $P^{\prime}$ is carried by $\psi^{-1}$ into $R$. So $\mu(R) \geq(1-\theta) \mu^{\prime}\left(P^{\prime}\right)$. But $\mu(R) \leq$ $(1+\varepsilon) B\left(\alpha_{0}+\varepsilon_{0}\right)^{2} t_{1}$, by proposition $4.3(\mathrm{c})$, while $\mu\left(P^{\prime}\right) \geq(1 /(1+\varepsilon)) B^{\prime} \alpha_{0}^{2} w_{1}$. Thus

$$
\frac{w_{1}}{t_{1}} \leq \frac{1}{1-\theta}(1-\varepsilon)^{2} \frac{B}{B^{\prime}}\left(1+\varepsilon_{0} / \alpha_{0}\right)^{2} .
$$

Choosing $\theta$ small, then $\varepsilon$ small, then $\alpha_{0}$ as small as required for this $\varepsilon$, then $\varepsilon_{0}$ very small, we get

concluding the lemma.

$$
\frac{w_{1}}{t_{1}} \leq B / B^{\prime}+\varepsilon_{1}=C+\varepsilon_{1}
$$


Now a function $T(x, t)$ will be defined as that unique $T$ (if it exists) for which $\psi\left(\bar{k}_{t} x\right)=\bar{k}_{T}^{\prime}(\psi(x)$.

(4.5) Lemma. For every $t \neq 0, T(x, t)$ is defined and $0<T(x, t) / t \leq C$ for a.e. $x$.

Proof. We show only for $t>0$; it is easy then to get the result for negative $t$ as well. Choose $t>0$. Now,

$$
\mu\left(g_{\rightarrow r} U_{e^{-r_{t}}}\right)=\mu\left(U_{e^{-r_{t}}}\right) \rightarrow 1 \quad \text { as } r \uparrow \infty,
$$

by lemma 4.4. So for a.e. $x$ there is a sequence $0<r_{1}<r_{2}<\cdots \infty$ with $x \in g_{-r_{m}} U_{e^{-r_{m t}}}$ for all $m=1,2, \ldots$, Let $x_{m}=g_{r_{m}} x, t_{m}=e^{-r_{m} t}, x^{\prime}=\psi(x), x_{m}^{\prime}=\psi\left(x_{m}\right)=g_{r_{m}}^{\prime} x^{\prime}$. Let $y=\bar{k}_{t} x, y_{m}=g_{r_{m}} y=\bar{k}_{t_{m}} x_{m}, y^{\prime}=\psi(y), y_{m}^{\prime}=\psi\left(y_{m}\right)=g_{r_{m}}^{\prime} y^{\prime}$. By definition of $U_{t_{m}}$, we have $y_{m}^{\prime}=g_{u_{m}}^{\prime} \bar{h}_{v_{m}}^{\prime} \bar{k}_{w_{m}}^{\prime} x_{m}^{\prime}$ with $\left|u_{m}\right|,\left|v_{m}\right|,\left|w_{m}\right| \rightarrow 0$ and $0<w_{m} / t_{m} \leq B / B^{\prime}+\varepsilon_{m}$, with $\varepsilon_{m} \rightarrow 0$, because of lemma 4.4.

Now:

$$
\begin{aligned}
& y_{1}^{\prime}=g_{r_{1}-r_{m}}^{\prime} y_{m}^{\prime}=g_{r_{1}-r_{m}}^{\prime} g_{u_{m}}^{\prime} \bar{h}_{v_{m}}^{\prime} \bar{k}_{w_{m}}^{\prime} x_{m}^{\prime}
\end{aligned}
$$

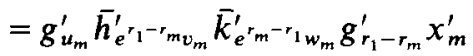

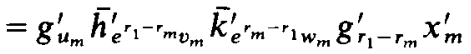

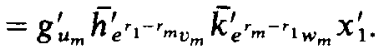

Note that $u_{m}$ and $e^{r_{1}-r_{m}} \rightarrow 0$, so $\bar{k}_{e^{r_{m}-r_{1}}}^{\prime} w_{m} x_{1}^{\prime} \rightarrow y_{1}^{\prime}$. Furthermore,

$$
\begin{aligned}
e^{r_{m}-r_{1}} w_{m} & =e^{r_{m}-r_{1}} \frac{w_{m}}{t_{m}} t_{m} \\
& \leq e^{r_{m}-r_{1}}\left(C+\varepsilon_{m}\right) t_{m} \\
& =\left(C+\varepsilon_{m}\right) e^{-r_{t}} t=\left(C+\varepsilon_{m}\right) t_{1} .
\end{aligned}
$$

Choose a convergent subsequence of $e^{r_{m}-r_{1}} w_{m}$ (since we see that it is bounded); it converges to $C_{0} t_{1}$, where $C_{0} \leq C ; C_{0}$ depends on lots of things, of course. Then $y_{1}^{\prime}=\bar{k}_{C_{0} t_{1}}^{\prime} x_{1}^{\prime}$, so, applying $g_{-r_{1}}^{\prime}, y^{\prime}=\bar{k}_{C_{0} t}^{\prime} x^{\prime}$, and $T(x, t)$ is defined and $\leq C t$, proving the lemma.

(4.6) LEMMA. $\psi$ is uniformly continuous on a set of measure 1.

Proof. By the previous lemma, for all $x$ in a set $N$ of measure 1 the set $V(x)$ of $t \neq 0$ for which $T(x, t) / t \leq C$ has full measure on $\mathbb{R}$ (use Fubini). We show that for any local cylinder $P$ in $M, \psi$ is uniformly continuous on $N \cap P$. Choose $x_{1}, x_{2}$ in $N \cap P$. There is some disk $D \subset P \cap M_{0}$ for which $D$ meets the LC segments of $P$ through $x_{1}$ and $x_{2}$ respectively in points $\bar{k}_{t_{1}} x_{1}=y_{1}$ and $\bar{k}_{t_{2}} x_{2}=y_{2}$ with $t_{i} \in V\left(x_{i}\right)$; this is because each $V\left(x_{i}\right)$ has full measure in $\mathbb{R}$, and the Poincaré map from segment to segment along disks is measure-preserving. Furthermore, $\left|t_{2}\right|$ may be made as small as desired. Now

$$
x_{2}=\bar{k}_{-t_{2}} y_{2}=\bar{k}_{-t_{2}} g_{r} \bar{h}_{s} y_{1}=\bar{k}_{-t_{2}} g_{r} \bar{h}_{s} \bar{k}_{t_{1}} x_{1}
$$

so setting $w_{i}=T\left(x_{i}, t_{i}\right)$, we have

$$
\psi\left(x_{2}\right)=\bar{k}_{-w_{2}}^{\prime} \psi\left(y_{2}\right)=\bar{k}_{-w_{2}}^{\prime} g_{r}^{\prime} \bar{h}_{s}^{\prime} \psi\left(y_{1}\right)=\bar{k}_{-w_{2}}^{\prime} g_{r} \bar{h}_{s} \bar{k}_{w_{1}} \psi\left(x_{1}\right) .
$$

Then measuring the distance of close points in $M$ by $d\left(g_{r} \bar{h}_{s} \bar{k}_{t} x, x\right)=\max (|r|,|s|,|t|)$, and analogously in $M^{\prime}$, and using the fact that $\left|w_{i}\right| \leq C\left|t_{i}\right|$ and that $\left|t_{2}\right|$ may be made 
arbitrarily small, we get

$$
d\left(\psi\left(x_{1}\right), \psi\left(x_{2}\right)\right) \leq C d\left(x_{1}, x_{2}\right),
$$

which proves the lemma.

To complete the proof of proposition 4.1, note that if the continuous extension of $\psi$ sends $x$ to $x^{\prime}$ then it sends $\bar{k}_{t} x$ to some $\bar{k}_{w}^{\prime} x^{\prime}$ with $0<w / t \leq C$, for every $t \neq 0$. Arguing similarly for $\psi^{-1}$, we have $0<t / w \leq 1 / C$ also, so $w=C t$.

(4.7) Corollary. If $(\mathrm{g}, M)$ and $\left(\mathrm{g}^{\prime}, M^{\prime}\right)$ are good normalized Anosov flows, and $\phi:\left(\bar{h}_{1}, \mu\right) \mapsto\left(\bar{h}_{1}^{\prime}, \mu^{\prime}\right)$ is a conjugacy between their (uniform) time 1 horocycle transformations, then there is a real number $s_{0}$ and a homeomorphic, orientation-preserving conjugacy $\theta:(M, g) \rightarrow\left(M^{\prime}, g^{\prime}\right)$ such that $\phi x=\bar{h}_{s_{0}}^{\prime} \theta x$ for $\mu$-a.e. $x$.

(4.8) Corollary. The set of lengths of closed orbits of $g$ is a conjugacy invariant of $\left(\bar{h}_{1}, \mu\right)$.

Recent work of A. Katok [K] based on ideas and work of Livčic [L] shows that, given an isomorphism of the fundamental groups of $S$ and $S^{\prime}$ which preserves the lengths of the unique closed geodesic in each (free) homotopy class, this isomorphism is induced by a homeomorphic conjugacy of $(g, U(S))$ and $\left(g^{\prime}, U\left(S^{\prime}\right)\right)$. Therefore (4.8) may be made more precise:

(4.9) COROLlary. The function on the fundamental group of $S$ which assigns to each (free) homotopy class the length of the unique closed geodesic in the class, is a complete conjugacy invariant for the ergodic transformation $\left(\overline{h_{1}}, \mu\right)$.

(4.10) CoRollary. Let $C\left(\bar{h}_{1}, \mu\right)$ be the set of invertible $\mu$-nonsingular transformation on $M$ which commute with $\bar{h}_{1}$. Then $C\left(\bar{h}_{1}, \mu\right) /\left\{\bar{h}_{s}: s \in \mathbb{R}\right\}$ is finite.

Proof. Let $\mathscr{G}$ be the group of those homeomorphisms of $\theta$ which commute with $g$, $\bar{h}$, and $\bar{k}$. Then each coset of $\{\bar{h}: s \in \mathbb{R}\}$ in $\mathscr{C}(\bar{h}, \mu)$ contains precisely one member of $\mathscr{G}$. We show that $\mathscr{G}$ is finite.

From the fact that the set $\left\{g_{r} \bar{h}_{s} \bar{k}_{t} x:|r|,|s|,|t|<1\right\}$ is an open neighbourhood of $x$, that the action of some $\mathscr{G}$ on this neighbourhood is completely determined by what it does to $x$, and that $M$ is compact, one easily gets that $\mathscr{G}$ is compact in the topology of uniform convergence on $M$.

If $\theta$ in $\mathscr{G}$ is uniformly within $\delta$ of the identity, then $\overline{h_{s}} \theta(x)=\theta\left(\bar{h}_{s} x\right)$ is within $\delta$ of $\bar{h}_{s} x$ for all $s$, and lemma 3.9 or corollary 3.10 tells us that $\theta(x)=\bar{h}_{S(x)} x$ for all $x$, provided $\delta$ is sufficiently small.

From $\theta \bar{h}=\bar{h} \theta$ we get $S\left(\bar{h}_{s} x\right)=S(x)$ for all $s$, so from ergodicity of $\bar{h}$ we get that $S$ is a.e. constant; and since $\theta$ is a homeomorphism, $S$ is everywhere constant. Then from $\theta g=g \theta$ and $g_{r} \bar{h}_{s}=\bar{h}_{e^{r}} g_{r}$ we see that this constant is zero.

Note that Ratner's proof of the above fact in the constant curvature case [R1] goes by explicitly describing $\mathscr{C}(\vec{h}, \mu)$.

(4.11) Problem (see [R3]). To what extent do Ratner's other results in [R1], [R2], [R3] hold here? In particular, what about the 'Factor Theorem' in [R2]? 
(4.12) Problem. Ratner's results actually hold for constant curvature surfaces of finite volume. Can our variable curvature results be generalized in some such way? Presumably uniform bounds on curvature would be needed. It would probably be necessary first to get a 'uniform parametrization' result and some 'almost unique ergodicity' as in Dani [D].

\section{Differentiability}

Now let us begin with a homeomorphic conjugacy $\theta$ from $(g, M)$ to $\left(g^{\prime}, M^{\prime}\right)$, with $g$ and $g^{\prime}$ normalized. An easy topological argument shows that such a homeomorphism must take the contracting foliation in $M$ to that in $M^{\prime}$, and similarly for the expanding foliation. Then uniqueness of the uniform parametrizations show that $\bar{h}$ is taken to $\bar{h}^{\prime}$ and $\bar{k}$ to $\bar{k}^{\prime}$, provided $\{g, \bar{h}, \bar{k}\}$ and $\left\{g, \bar{h}^{\prime}, \bar{k}^{\prime}\right\}$ are properly oriented and properly scaled.

Returning to the notation of $\S 2$, we also have the smooth flows of $h$ and $k$ on $M$, with infinitesimal generators $H$ and $K$, and similarly $h^{\prime}$ and $k^{\prime}$ on $M^{\prime}$ with infinitesimal generators $H^{\prime}, K^{\prime}$. Recall also the functions $\rho$ and $\rho^{\prime}$, defined near $M \times(0,0)$ and $\rho^{\prime}$ defined near $M^{\prime} \times(0,0)$. Define $S$ and $T$ by $h_{S(x, s)}^{\prime}(\theta x)=\theta\left(h_{s} x\right)$ and $k_{T(x, t)}^{\prime}(\theta x)=\theta\left(k_{t} x\right)$.

(5.1) LEMMA. $s \mapsto S(\cdot, s)$ and $t \mapsto T(\cdot, t)$ are continuously differentiable (as functions with values in $\left.C^{0}\left(M^{\prime}\right)\right)$.

Proof. $\rho(x, s, t)=\rho^{\prime}(\theta x, S(x, s), T(x, t))$. But $\rho(\cdot, s, t) / s t \rightarrow a \quad$ as $\quad s, t \rightarrow 0$, and $\rho^{\prime}(\cdot, s, t) / s^{\prime} t^{\prime} \rightarrow a^{\prime}$ as $s^{\prime}, t^{\prime} \rightarrow 0$ (both uniformly on $M$ ). Also, $S(\cdot, s) \rightarrow 0$ uniformly as $s \rightarrow 0$ and $T(\cdot, s) \rightarrow 0$ uniformly as $t \rightarrow 0$. Choose $\delta>0$ so small that if $|s|,|t|<\delta$ then $S(\cdot, s) T(\cdot, t) / s t=(1 \pm \varepsilon)\left(a / a^{\prime} \circ \theta\right)$ then

$$
\log \frac{S(\cdot, s)}{s}+\log \frac{T(\cdot, t)}{t}=\varepsilon_{1}+\log \frac{a}{a^{\prime} \circ \theta},
$$

where $\varepsilon_{1}=\log (1 \pm \varepsilon)$. Fixing $t$ with $0<|t|<\delta$, we see that $|s|<\delta \rightarrow \log (S(\cdot, s) / s)$ is within $\varepsilon_{1}$ of a continuous function. Since $\varepsilon_{1}$ can be made arbitrarily small, $\log (S(\cdot, s) / s) \rightarrow$ a continuous function, uniformly as $s \rightarrow 0$. So, $S(\cdot, s) s \rightarrow$ a non-zero continious function, uniformly, as $s \rightarrow 0$. Thus $s \mapsto S(\cdot, s)$ is differentiable at $s=0$, as a function with values in $C^{0}\left(M^{\prime}\right)$. Now: $S\left(x, s_{0}+s\right)=S\left(x, s_{0}\right)+S\left(h_{s_{0}} x, s\right)$, so $s \mapsto S(\cdot, s)$ is differentiable at all $s$. Furthermore,

$$
\left.\frac{d S(\cdot, s)}{d s}\right|_{s=s_{0}}=\left.\frac{d S\left(h_{s_{0}} \cdot, s\right)}{d s}\right|_{s=0} .
$$

Examining the previous argument more carefully,

$$
\frac{S\left(h_{s_{0}}, s\right) T\left(h_{s_{0}} \cdot t\right)}{s t}=(1 \pm \varepsilon) \frac{a\left(h_{s_{0}} \cdot\right)}{a^{\prime} \circ \theta\left(h_{s} \cdot\right)},
$$

so

$$
\log \left(\frac{S\left(h_{s_{0}} \cdot s\right)}{s}\right)+\log \left(\frac{T\left(h_{s_{0}} \cdot t\right)}{t}\right)=\varepsilon_{1}+\log \frac{c\left(h_{s_{0}} \cdot\right)}{c^{\prime} \circ \theta\left(h_{s_{0}} \cdot\right)}
$$


and therefore $S\left(h_{s_{0}} x, s\right) / s$ is, as a function of both $s_{0}$ and $x$, close to a non-vanishing continuous function of $s$ and $x$. Thus $s \mapsto S(\cdot, s)$ is a continuously differentiable function from $s$ to $C^{0}\left(M^{\prime}\right)$. Similar arguments work for $t \mapsto T(\cdot, t)$.

(5.2) Theorem. $\theta$ is a $C^{1}$ diffeomorphism.

Proof. By symmetry, it suffices to show that $\theta$ is $C^{1}$. Let $x^{\prime}=\theta(x)$, and let $\theta\left(k_{t} h_{s} g_{r} x\right)=$ $k_{t^{\prime}}^{\prime} h_{s^{\prime}}^{\prime} g_{r^{\prime}}^{\prime} x^{\prime}$. Then $r=r^{\prime}, S^{\prime}=S\left(g_{r} x, s\right)$, and $t^{\prime}=T\left(h_{s} g_{r} x, t\right)$. Since $(r, s, t)$ and $\left(r^{\prime}, s^{\prime}, t^{\prime}\right)$ give local $C^{1}$ coordinates near $x$ and $x^{\prime}$ respectively, it suffices to show that for fixed $x$ the map

$$
(r, s, t) \mapsto\left(r^{\prime}, s^{\prime}, t^{\prime}\right)=\left(r, S\left(g_{r} x, s\right), T\left(h_{s} g_{r} x, t\right)\right)
$$

is $C^{1}$. We do this by writing $S\left(g_{r} x, s\right)$ and $T\left(h, g_{r} x, t\right)$ in another way which makes it obvious.

Let $\alpha$ be defined on $M \times \mathbb{R} \times \mathbb{R}$ by $g_{r} h_{s} x=h_{\alpha(x, s, r)} g_{r} x$. Then $\alpha(\cdot, \cdot, r)$ is, for each $r$, a $C^{1}$ reparametrization of $h$. Let $\tilde{\alpha}$ be the inverse reparametrization: $\tilde{\alpha}(x, \alpha(x, s, r), r)=s . \tilde{\alpha}$ is likewise $C^{1}$. Similarly define $\alpha^{\prime}$ and $\tilde{\alpha}^{\prime}$ on $M^{\prime} \times \mathbb{R} \times \mathbb{R}$.
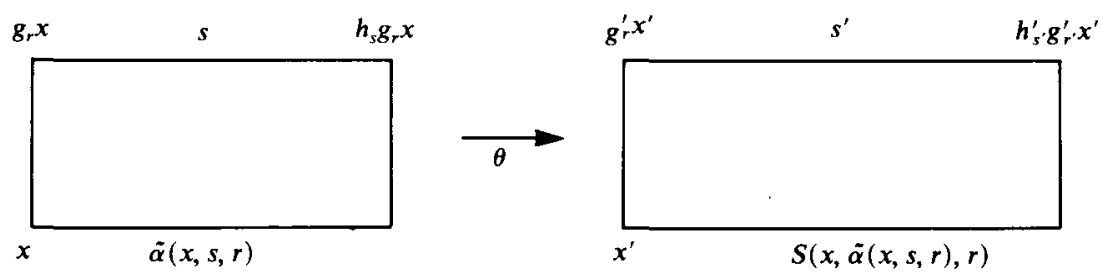

Figure 7

Guided by figure 7 , we see that $s^{\prime}=S\left(g_{r} x, s\right)$ may also be written as $\alpha^{\prime}\left(x^{\prime}, S(x, \tilde{\alpha}(x, s, r)), r\right)$. If $x$ is fixed so is $x^{\prime}$. The map $(s, r) \mapsto \tilde{\alpha}(x, s, r) \mapsto$ $S(x, \tilde{\alpha}(x, s, r), r)$ is $C^{1}$, using lemma 5.1. Plugging this into

$$
\alpha^{\prime}\left(x^{\prime}, S(x, \tilde{\alpha}(x, s, r), r), r\right)
$$

gives that $(s, r) \mapsto s^{\prime}=S\left(g_{r} x, s\right)$ is $C^{1}$ for fixed $x$.

It remains to show that $t^{\prime}=T\left(h_{s} g_{r} x, t\right)$ is a $C^{1}$ function of $r, s, t$. The argument is similar. We will need the function $\tau$ from $\S 2$, and the analogous function $\tau^{\prime}$. These are $C^{1}$ where defined. The function $\tau(x, s, \cdot)$ is a reparametrization defined near 0 , and we denote by $\tilde{\tau}$ its local inverse: $\tilde{\tau}(x, s, \tau(x, s, t))=t$. Similarly, $\tilde{\tau}^{\prime}$.
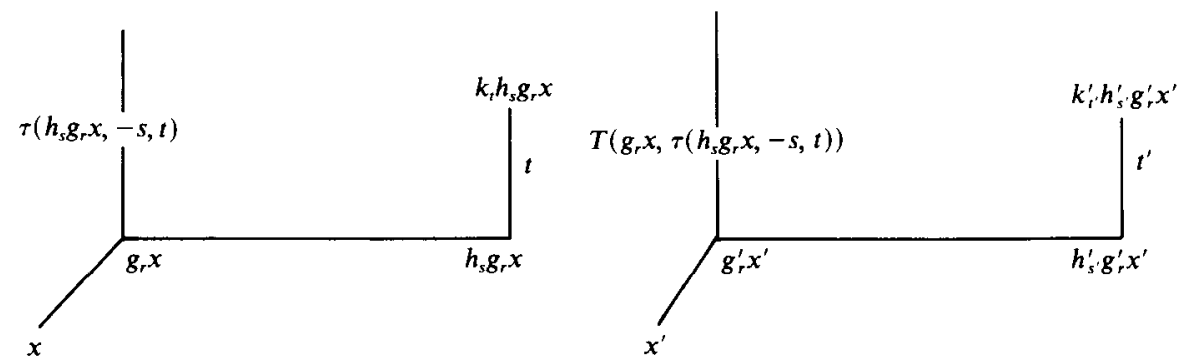

Figure 8 
Guided by figure 8 , we see that

$$
t^{\prime}=\tilde{\tau}^{\prime}\left(h_{s^{\prime}}^{\prime} g_{r}^{\prime} x^{\prime},-s, T\left(g_{r} x, \tau\left(h_{s} g_{r} x,-s, t\right)\right)\right) .
$$

Now we have already shown that for fixed $x,(r, s) \mapsto S\left(g_{r} x, s\right)$ is $C^{1}$, so $(r, s) \mapsto$ $T\left(g_{r} x, t\right)$ is $C^{1}$, by the same argument. Then application of the chain rule gives the result.

\section{The isometry question}

Let $S$ and $S^{\prime}$ again be compact orientable surfaces of negative curvature. Any isometry from $S$ to $S^{\prime}$ lifts to a homeomorphic conjugacy of $(g, U(S))$ with $\left(g^{\prime}, U\left(S^{\prime}\right)\right)$. We may then compose this with some fixed $g_{r}^{\prime}$, and the result is again a homeomorphic conjugacy of $(g, U(S))$ with $\left(g^{\prime}, U\left(S^{\prime}\right)\right)$. Does every homeomorphic conjugacy of $(g, U(S))$ with $\left(g^{\prime}, U\left(S^{\prime}\right)\right)$ arise in this manner? We cannot answer this question, but there are two relevant comments which will be made.

There is a unique Riemannian metric on the tangent bundle which projects to the Riemannian metric on the surface, whose fibres have their natural arclength, and the natural circle action is by isometries; see [B].

(6.1) THEOREM (A. Weinstein). The following statements are equivalent for a homeomorphic conjugacy $\theta:(\mathrm{g}, U(S)) \rightarrow\left(\mathrm{g}^{\prime}, U\left(S^{\prime}\right)\right)$.

(a) $\theta$ is an isometry for the canonical metrics.

(b) $\theta$ takes a fibre in $U(S)$ to a fibre in $U\left(S^{\prime}\right)$.

(c) $\theta$ is the lift of an isometry: $S \rightarrow S$.

Proof. (a) $\Rightarrow$ (b) The group $\mathscr{I}$ of isometries of $U(S)$ is a compact Lie group in, say, the topology of uniform convergence. Its component $\mathscr{I}_{0}$ of the identity is therefore a compact connected Lie group. Let $\mathscr{T}$ be the subgroup of $\mathscr{Y}_{0}$ given by the canonical circle action on $U(S)$.

(6.2) LeMMA. $\mathscr{T}=\mathscr{I}_{0}$.

Proof. $\mathscr{T}$ is a maximal torus in $\mathscr{I}_{0}$, for if there were a two-dimensional toral subgroup of $\mathscr{I}_{0}$ containing $\mathscr{T}$, then the action of $\mathscr{T}$ could be factored out to get an action of a 1-torus on $S$, whereas it is well-known (see [Y-B]) that the isometries of $S$ are discrete.

The only compact connected Lie groups with a maximal torus of dimension 1 are $\mathbb{T}^{1}$, SU(2), and $S O(3)$, so $\mathscr{I}_{0}$ is isomorphic to one of these. We shall show that the second and third alternatives are impossible.

Consider an orbit of the action of $\mathscr{I}_{0}$. It must have dimension $\geq 1$, since the orbits under $\mathscr{T}$ are circles. If an orbit had dimension 2 then it would be a 2 -dimensional homogeneous space of $\mathrm{SU}(2)$ or $\mathrm{SO}(3)$, hence it would be the 2-sphere or the projective plane. $\mathscr{T}$ acts on the orbit without fixed points, which would be impossible here, by the Lefschetz fixed point theorem, so dimension 2 is ruled out.

If an orbit had dimension 3 then the orbit would be a 3-dimensional compact submanifold of $U(S)$, hence would be all of $U(S)$. Thus $U(S)$ would be a quotient of $\mathrm{SU}(2)$ or $\mathrm{SO}(3)$ by a discrete subgroup, so $\mathrm{SO}(3)$ would have $\mathrm{SU}(2)$ or $\mathrm{SO}(3)$ 
as a simply connected covering space. But the simply connected covering space of $U(S)$ is in fact $\mathbb{R}^{3}$, giving a contradiction, which proves the lemma.

(a) $\Leftarrow(b)$ : If $\theta$ is an isometry, we may get another free isometric circle action on $U(S)$ by using $\theta$ to carry over to $U(S)$ the circle action on $U\left(S^{\prime}\right)$. By the lemma, this action must have the same orbit as $\mathscr{T}$; thus $\psi$ carries fibres to fibres.

(b) $\Rightarrow$ (c): If $\theta$ sends fibres to fibres, then the map $\theta$ may be pushed down to a map: $S \rightarrow S^{\prime}$, and the pushdown sends geodesics to geodesics in a length-preserving way; thus the pushdown is an isometry.

(c) $\Rightarrow$ (a) because the construction of the metric on $U(S)$ and $U\left(S^{\prime}\right)$ is functorial.

Finally, we quote the following uniqueness result, proved by J. Smillie; the proof will probably appear elsewhere.

(6.2) THEOREM (J. Smillie). If $\theta_{1}$ and $\theta_{2}$ are isometries $U(S) \rightarrow U\left(S^{\prime}\right)$, and they have the same effect on the homotopy groups, then $\theta_{2}=g_{r_{0}}^{\prime} \circ \theta_{1}$ for some fixed $r_{0}$.

This still leaves us with the question:

(6.3) Is every homeomorphic conjugacy from $(g, U(S))$ to $\left(g^{\prime}, U\left(S^{\prime}\right)\right)$ a geodesic translate of a lift of an isometry: $S \rightarrow S^{\prime}$ ?

In view of the aforementioned result of Katok (see (4.11), (4.12)), this is equivalent to the following well-known question (see, for example, Guillemin-Kazhdan [G-K] and Ballman et al. [B-B]).

(6.4) Given an isomorphism of the fundamental groups of $S$ and $S^{\prime}$ which preserves the length of the unique closed geodesic in each (free) homotopy class, is it induced by an isometry of $S$ with $S^{\prime}$ ?

There are two related special situations in which the answer to (6.4) is affirmative. The arguments make use of the following result of A. Katok [K]. Since his proof is short and sweet it will be included.

(6.5) Proposition. Let $S$ be a compact surface of negative curvature, and $\Gamma(S)$ its fundamental group. Let $l$ be the function on $\Gamma(S)$ which assigns to $\gamma$ the length of the unique closed geodesic isotopic to the members of $\gamma$. Suppose $\kappa$ is a positive function on $S$ so that the conformally equivalent metric obtained by scaling by $\kappa$ has the same length function on $\Gamma(S)$. Then $\kappa \equiv 1$.

Proof. Let $V$ be the surface area for the original metric, and $V^{\prime}$ the surface area in the new metric. We shall show that $\kappa \neq 1 \Rightarrow V^{\prime}>V$. This will do it, since the situation is actually symmetric. Now, the Schwarz inequality tells us that if $\kappa \not \equiv 1$ then

$$
\iint \sqrt{\kappa} d A \leq \theta \sqrt{\iint_{S} \kappa d A} \sqrt{\iint_{S} d A}, \quad \text { where } 0 \leq \theta<1 .
$$

Choose a closed geodesic $C$ isotopic to the members of $\gamma$, and approximately uniformly distributed (with respect to the original metric). Then $l(\gamma) \leq$ new length 
of $C=\int \sqrt{\kappa} d s$, so:

$$
1 \leq \frac{1}{l(\gamma)} \int_{C} \sqrt{\kappa} d s \approx \frac{1}{V} \iint_{S} \sqrt{\kappa} d A \leq \frac{1}{V} \theta \sqrt{\iint_{S} \sqrt{\kappa} d A} \sqrt{\iint_{S} d A}=\theta \frac{\sqrt{V^{\prime}}}{\sqrt{V}} .
$$

(6.6) COROLlARY. Let $S$ be a compact surface of negative curvature such that its length function $l$ on $\Gamma(S)$ has multiplicity one, i.e. $\gamma \neq \gamma^{\prime} \Rightarrow l(\gamma) \neq l\left(\gamma^{\prime}\right)$. Then there is some $\delta>0$ so that if $\kappa_{1}, \kappa_{2}$ are $C^{2}$ positive functions $S$ with $\left|\kappa_{i}-1\right|<\delta$, and $l_{1}, l_{2}$ the length functions on $\Gamma(S)$ obtained by using the $\kappa_{i}$ to make conformally equivalent metrics on $S$, and $\alpha$ is an isomorphism of $\Gamma(S)$ carrying $l_{1}$ to $l_{2}$, then $\alpha=$ identity and $\kappa_{1}=\kappa_{2}$.

Proof. The values of $l$ form a discrete set. Let $\Gamma_{0}$ be a finite set of generators for $\Gamma(S)$. Then there is some $\varepsilon>0$ such that if $\left|\left(l(\gamma) / l\left(\gamma^{\prime}\right)\right)-1\right|<\varepsilon$, for some $\gamma$ in $\Gamma_{0}$ and any $\gamma^{\prime}$ in $\Gamma(S)$, then $\gamma=\gamma^{\prime}$. Now choose $\delta$ so small that

$$
\frac{l_{2}(\gamma)}{l_{1}(\alpha(\gamma))}=1 \Rightarrow\left|\frac{l_{1}(\gamma)}{l(\alpha(\gamma))}-1\right|<\varepsilon .
$$

This can be done, since $l_{1}(\gamma) / l(\gamma)$ and $l_{2}(\gamma) / l(\gamma)$ will be close to 1 , uniformly in $\gamma \in \Gamma(S)$, if $\delta$ is small. Thus: $\gamma \in \Gamma_{0} \Rightarrow \gamma=\alpha(\gamma) \Rightarrow \alpha=$ identity and $l_{1}(\gamma)=l_{2}(\gamma)$ for all $\gamma \in \Gamma(S)$, and by Katok's result, $\kappa_{1}=\kappa_{2}$.

(6.7) CoRollary. Let $S$ be a compact surface of constant negative curvature. Then there is some $\delta>0$ so that if $\kappa_{1}, \kappa_{2}$ are $C^{2}$ positive functions on $S$ with $\left|\kappa_{i}-1\right|<\delta$, and $l_{1}, l_{2}$ the corresponding length functions on $\Gamma(S)$, obtained by the associated conformally equivalent metrics, and $\alpha$ is an isomorphism of $\Gamma(S)$ carrying $l_{1}$ to $l_{2}$, then there is an isometry $i$ of $S$ carrying $\kappa_{1}$ to $\kappa_{2}$.

Proof. There is a finite subset $\Gamma_{0}$ of $\Gamma(S)$ such that if $l(\alpha(\gamma))=l(\gamma)$ for all $\gamma \in \Gamma_{0}$ then $l(\alpha(\gamma))=l(\gamma)$ for all $\gamma \in \Gamma(S)$. This is a folk-theorem of hyperbolic geometry, which was explained to one of us by L. Bers. If $\delta$ is small enough, then

$$
\begin{aligned}
l_{2}(\gamma)=l_{1}(\alpha(\gamma)) \text { for all } \gamma \in \Gamma_{0} & \Rightarrow l_{2}(\gamma)=l_{1}(\alpha(\gamma)) \text { for all } \gamma \in \Gamma(S) \\
& \Rightarrow \text { there is an isometry } i \text { of } S \text { such that } \alpha(\gamma)=\gamma \circ i
\end{aligned}
$$

Then $l_{2}(\gamma)=l_{1}(\gamma \cdot i)$, so by Katok's result, $\kappa_{2}=\kappa_{1} \circ i$.

\section{REFERENCES}

[A] D. V. Anosov. Geodesic flows on closed Riemannian manifolds with negative curvature. Proc. Steklov Inst. of Math. \#90 (1967) (AMS translation, 1969).

[B] M. Berger (A. L. Besse). Manifolds All of Whose Geodesics Are Closed. Springer-Verlag, 1978.

[B-B] W. Ballman, M. Brin, K. Burns, P. Eberlein, A. Katok \& R. Osserman. Manifolds of nonpositive curvature. MSRI preprint, 1984.

[B-M] R. Bowen \& B. Marcus. Unique ergodicity for horocyclic foliations. Israel J. Math. 43 (1977), 43-67.

[D] S. G. Dani. Dynamics of the horospherical flow. Bull. Amer. Math. Soc. 3 (1980), 1037-1039.

[G-K] V. Guillemin \& D. Kazhdan. Some inverse spectral results for negatively curved 2-manifolds. Topology 19 (1980), 301-302.

[H-P] M. Hirsch \& C. Pugh. Smoothness of horocycle foliations. J. Diff. Geom. 10 (1975), 225-238. 
[K] A. Katok. Closed geodesics and conjugacy of geodesic flows. In preparation.

[L] A. N. Livčic. Some homology properties of U-systems. Mat. Zametki 10 (1971), 555-564; Math. Notes 10 (1971), 758-763.

[M1] B. Marcus. Unique ergodicity of the horocycle flow: variable negative curvature case. Israel J. Math. 21 (1975), 133-144.

[M2] B. Marcus. Topological conjugacy of horocycle flows. Amer. J. Math. (1983), 623-632.

[Ma] G. A. Margulis. Certain measures associated with U-flows on compact manifolds. Funct. Anal. Appl. 4 (1970), 55-67.

[P] J. F. Plante. Anosov flows. Amer. Jour. Math. XCIV (1972), 729-754.

[R1] M. Ratner. Rigidity of horocycle flows. Ann. Math. 115 (1982), 587-614.

[R2] M. Ratner. Factors of horocycle flows. Ergod. Th. \& Dynam. Sys. 2 (1982), 465-489.

[R3] M. Ratner. Horocycle flows; joinings and rigidity of products. Ann. Math. 118 (1983), 277-313.

[R4] M. Ratner. Ergodic theory in hyperbolic space. In Conference in Modern Analysis and Probability, Contemporary Math. 26. Amer. Math. Soc.

[R5] M. Ratner. Rigidity of time changes for horocycle flows. To appear.

[Y-B] K. Yano \& S. Bochner. Curvature and Betti Numbers. Princeton University Press, Princeton, NJ, 1953. 